\title{
The Turbulence Cascade in the Near Wake of a Square Prism
}

\author{
F. Alves Portela ${ }^{1} \dagger$, G. Papadakis ${ }^{1}$ and J. C. Vassilicos ${ }^{1}$ \\ ${ }^{1}$ Turbulence, Mixing and Flow Control Group, Department of Aeronautics, Imperial College \\ London, London SW7 2AZ, UK
}

(Received xx; revised xx; accepted xx)

We present a study of the turbulence cascade on the centreline of an inhomogeneous and anisotropic near field turbulent wake generated by a square prism at $R e=3900$ using the Kármán-Howarth-Monin-Hill (KHMH) equation. This is the fully generalised scale-by-scale energy balance which, unlike the Kármán-Howarth equation, does not require homogeneity or isotropy assumptions. Our data are obtained from a direct numerical simulation (DNS) and therefore enable us to access all the processes involved in this energy balance. A significant range of length-scales exists where the orientation averaged non-linear interscale transfer rate is approximately constant and negative, indicating a forward turbulence cascade on average. This average cascade consists of coexisting forward and inverse cascade behaviours in different scale space orientations. With increasing distance from the prism but within the near field of the wake, the orientationaveraged non-linear interscale transfer rate tends to be approximately equal to minus the turbulence dissipation rate even though all the inhomogeneity-related energy processes in the scale-by-scale energy balance are significant, if not equally important. We also find well-defined near $-5 / 3$ energy spectra in the streamwise direction, in particular at a centreline position where the inverse cascade behaviour occurs for streamwise oriented length-scales.

Key words:

\section{Introduction}

Kolmogorov's equilibrium cascade theory (Kolmogorov 1941a, $b, c$ ) applies to locally homogeneous turbulence and is perhaps the one firm theoretical pillar underpinning much of our understanding of turbulent flows as well as much of one-point (e.g. RANS) and two-point (e.g. LES) modelling of such flows over the past 75 years. However, recent developments have shown that this theory does not apply in, at least, extended regions of many turbulent flows where the theory might have been expected to be valid given that local statistical homogeneity is present. Examples of such flows are unsteady periodic turbulence (Goto \& Vassilicos 2015, 2016), grid-generated decaying turbulence (Vassilicos 2015), self-similar axisymmetric turbulent wakes (Vassilicos 2015; Castro 2016; Obligado et al. 2016, and references therein) and the outer region of turbulent boundary layers (Nedić et al. 2017). Even so, as Kraichnan (1974) already stated more than forty years ago, "Kolmogorov's 1941 theory has achieved an embarrassment of success. The $-5 / 3$-spectrum has been found not only where it reasonably could be expected but also

$\dagger$ Email address for correspondence: f.alves-portela13@ic.ac.uk; j.c.vassilicos@ic.ac.uk 
at Reynolds numbers too small for a distinct inertial range to exist and in boundary layers and shear flows where there are substantial departures from isotropy, and such strong effects from the mean shearing motion that the step-wise cascade appealed to by Kolmogorov is dubious". Kraichnan was referring to the Kolmogorov-Obukhov prediction (Obukhov 1941) that the turbulence energy spectrum is proportional to the $-5 / 3$ power of wavenumber in the inertial range. This particular prediction has indeed been an "embarrassment of success" because it is present even more widely than Kraichnan stated: it can indeed be found in relatively low Reynolds number flows, boundary layers, wakes, homogeneous shear flows, atmospheric turbulence and mixing layers (see e.g. Klebanoff 1955; Uberoi \& Frevmuth 1969; Champagne et al. 1970; Rogers \& Moser 1994; Ong \& Wallace 1996; Kravchenko \& Moin 2000; Wissink \& Rodi 2008); but it can also be found in those flow regions mentioned above where the Kolmogorov equilibrium is now known to be absent; and it can even be found in flow regions such as in the very inhomogeneous and anisotropic very near field production region of gridgenerated turbulence where it cannot be expected to apply from the very outset (see Gomes-Fernandes et al. 2015).

Even more fundamental than the $-5 / 3$ power law scaling of the energy spectrum is Kolmogorov's prediction that, in the inertial range of scales, the interscale energy transfer rate $\Pi$ is constant, i.e. independent of length-scale and viscosity $\nu$, and equal to $-\varepsilon$ where $\varepsilon$ is the turbulence dissipation rate per unit mass. There are various ways to derive the energy spectrum's $-5 / 3$ power law shape in the inertial range (see, for example, the textbooks by Tennekes \& Lumley 1972; Frisch 1995; Pope 2000; Mathieu \& Scott 2000; Lesieur 2008) but unlike the constancy of $\Pi$, no way is known which derives it directly from the Navier-Stokes equations without closure assumptions (Kraichnan 1974; Leslie 1973; McComb 2014). A straightforward dimensional analysis is typically carried out which starts from the premise that the energy spectrum depends only on $\varepsilon$ and wavenumber in the inertial range, a premise which can be justified by the result, itself obtained from Navier-Stokes, that $\Pi \approx-\varepsilon$ in the inertial range. If the energy spectrum is a one-dimensional spectrum (as typically measured in laboratory experiments) then an assumption of small-scale isotropy is also required (Tennekes \& Lumley 1972; Frisch 1995; Pope 2000; Mathieu \& Scott 2000; Lesieur 2008).

Whilst recent Direct Numerical Simulations (DNS) (Goto \& Vassilicos 2016) have shown that, in freely decaying periodic turbulence, $\Pi$ is neither constant nor equal to $-\varepsilon$ even though a $-5 / 3$ power law scaling of the energy spectrum is clearly present, Particle Image Velocimetry (PIV) measurements by Gomes-Fernandes et al. (2015) suggest that in the very near field of grid-generated turbulence where the turbulence is very inhomogeneous (even locally), anisotropic and building up, $\Pi / \varepsilon$ may be approximately independent of scale over a significant range. Adding to Kraichnan's "embarrassment of success", Gomes-Fernandes et al. (2015) find that the energy spectrum has a very welldefined $-5 / 3$ power law dependence on frequency in this region, a result also obtained and discussed in Laizet et al. (2013), Laizet et al. (2015) and Melina et al. (2016).

The approach taken by Gomes-Fernandes et al. (2015) relies on the Kármán-HowarthMonin-Hill (KHMH) equation: this is the fully generalised form of the Kármán-Howarth or Kármán-Howarth-Monin equation (see Frisch 1995) derived by Hill (2002a) which is valid under no assumptions whatsoever, i.e. irrespective of degrees of inhomogeneity, anisotropy and unsteadiness. The KHMH equation is an energy balance for the energy associated with eddies of a certain size. It takes into account both interscale and interspace transfers as well as advection, turbulence dissipation and production. This equation is the only rigorous way to make statements concerning $\Pi$ and $\varepsilon$ in a highly inhomogeneous and anisotropic region of a turbulent flow such as the very near-field grid- 
generated turbulence studied by Gomes-Fernandes et al. (2015). These authors used a planar two-component PIV to compute the various terms of this equation and therefore had to rely on a number of assumptions to obtain some of these terms. It is therefore essential to carry out a similar study in a well-resolved computational setting where all the terms of the KHMH equation can be calculated without having to resort to any unjustified assumption.

In this paper, without resorting to any assumption, we calculate every term of the $\mathrm{KHMH}$ equation in a DNS of a turbulent planar wake generated by a square prism and study its near field up to about $10 d$, where $d$ is the side of the square. We study the near wake of a square prism because $(i)$ it is numerically less demanding than a DNS of the near field of grid-generated turbulence and is nevertheless a significantly inhomogeneous and anisotropic flow; (ii) power law energy spectra have already been reported in the very near field of planar wakes (Ong \& Wallace 1996; Braza et al. 2006); and (iii) hot wire anemometry studies of highly reduced forms of the KHMH equation have already been carried out in turbulent planar wakes by Thiesset et al. $(2011 a)$, Thiesset et al. (2011b), Thiesset et al. (2013a) and Thiesset et al. (2014). This approach can give us the opportunity to both confirm and extend the results of Gomes-Fernandes et al. (2015) and Thiesset et al. (2013a). However we go beyond this indeed important and necessary confirmation and extension and offer some new insights concerning the various terms of the $\mathrm{KHMH}$ equation and the processes they represent.

In section 2 we introduce the KHMH equation and our DNS. In section 3 we examine energy spectra, second and third order structure functions and the Taylor frozen turbulence hypothesis. Measurements are often made at a point in time and the Taylor hypothesis is typically invoked to interpret a measured frequency spectrum in terms of a wavenumber spectrum. The wavenumber spectrum is related to the second order structure function by a Fourier transform and the scaling of the second order structure function follows from that of the third order structure function if the skewness which can be constructed from these two structure functions is independent of scale (see Pope 2000). In turn, the scaling of the third order structure function may result from the properties of the interscale energy transfer rate $\Pi$ and small-scale isotropy. Small-scale isotropy is discussed in both sections 3 and 4 . Section 4 offers a detailed analysis of each term of the KHMH equation at various positions along the near-wake's centreline. We conclude in section 5 .

\section{Methodology and Validation}

\subsection{The Generalised Scale by Scale Energy Budget}

The most general forms of scale-by-scale energy budget for incompressible turbulent flows have been derived without making any assumption about the nature of the turbulence by Duchon \& Robert (1999) without averaging and by Hill (1997), Hill (2001) and Hill (2002a) with averaging. Using the Reynolds decomposition (capital letters and 〈... indicate ensemble- and/or time-averaged quantities), the equation derived by Hill (1997), Hill (2001) and Hill (2002a) (which we refer to as KHMH equation) takes the form (see also Danaila et al. 2012) 


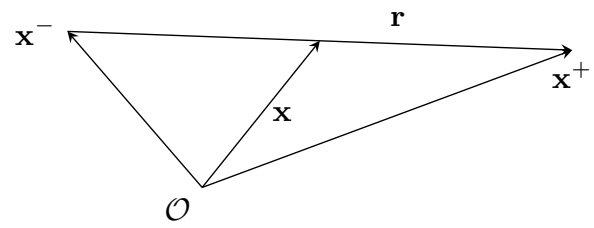

Figure 1: Illustration of the spatial position vectors $\mathbf{x}=\left(x_{1}, x_{2}, x_{3}\right), \mathbf{x}^{+}=\left(x_{1}^{+}, x_{2}^{+}, x_{3}^{+}\right)$ and $\mathbf{x}^{-}=\left(x_{1}^{-}, x_{2}^{-}, x_{3}^{-}\right)$in a reference frame centred on $\mathcal{O}$, and of separation vector $\mathbf{r}=\left(r_{1}, r_{2}, r_{3}\right)$.

$$
\begin{aligned}
& \frac{\partial\left\langle\delta q^{2}\right\rangle}{\partial t}+\frac{\partial \frac{U_{i}^{+}+U_{i}^{-}}{2}\left\langle\delta q^{2}\right\rangle}{\partial x_{i}}+\frac{\partial\left\langle\delta u_{i} \delta q^{2}\right\rangle}{\partial r_{i}}+\frac{\partial \delta U_{i}\left\langle\delta q^{2}\right\rangle}{\partial r_{i}}=-2\left\langle\delta u_{i} \delta u_{j}\right\rangle \frac{\partial \delta U_{j}}{\partial r_{i}}- \\
& -\left\langle\left(u_{i}^{+}+u_{i}^{-}\right) \delta u_{j}\right\rangle \frac{\partial \delta U_{j}}{\partial x_{i}}-\frac{\partial\left\langle\frac{u_{i}^{+}+u_{i}^{-}}{2} \delta q^{2}\right\rangle}{\partial x_{i}}-2 \frac{\partial\left\langle\delta u_{i} \delta p\right\rangle}{\partial x_{i}}+\nu \frac{1}{2} \frac{\partial^{2}\left\langle\delta q^{2}\right\rangle}{\partial x_{i} \partial x_{i}}+ \\
& +2 \nu \frac{\partial^{2}\left\langle\delta q^{2}\right\rangle}{\partial r_{i} \partial r_{i}}-4 \nu\left(\left\langle\frac{\partial \delta u_{j}}{\partial x_{i}} \frac{\partial \delta u_{j}}{\partial x_{i}}\right\rangle+\frac{1}{4}\left\langle\frac{\partial \delta u_{j}}{\partial r_{i}} \frac{\partial \delta u_{j}}{\partial r_{i}}\right\rangle\right)
\end{aligned}
$$

where $\delta q^{2}=\delta u_{i} \delta u_{i}$ in terms of the fluctuating velocity differences $\delta u_{i}=u_{i}^{+}-u_{i}^{-}$(for components $i=1,2,3), \delta U_{i}=U_{i}^{+}-U_{i}^{-}$where $U_{i}$ is a mean flow velocity component, $\delta p=p^{+}-p^{-}$where $p$ is fluctuating pressure, and the superscripts + and - distinguish quantities evaluated at $x_{i}+r_{i} / 2$ and $x_{i}-r_{i} / 2$, respectively, as illustrated in fig. 1 . Equation (2.1) is written in a six-dimensional reference frame $x_{i}, r_{i}$ where coordinates $x_{i}$ are associated with a location in physical space and the scale space is the space of all separations and orientations $\mathbf{r}=\left(r_{1}, r_{2}, r_{3}\right)$ between two-points (we refer to $r=|\mathbf{r}|$ as a scale). Throughout this paper, in the context of two-point statistics, we refer to isotropy as the independence of a given quantity on the orientation of the separation vector $\mathbf{r}$.

We follow Valente \& Vassilicos (2015) and Gomes-Fernandes et al. (2015) in the way we identify the terms in (2.1) as

$$
\mathcal{A}_{t}=-\mathcal{A}-\Pi-\Pi_{U}+\mathcal{P}+\mathcal{T}_{u}+\mathcal{T}_{p}+\mathcal{D}_{x}+\mathcal{D}_{r}-\varepsilon_{r}
$$

where each term is associated with a physical process in the budget of $\left\langle\delta q^{2}\right\rangle$ :

- $4 \mathcal{A}_{t}=\frac{\partial\left\langle\delta q^{2}\right\rangle}{\partial t}$ represents the rate of change in time of $\left\langle\delta q^{2}\right\rangle$ at a given physical point $x_{i}$ and separation $r_{i}$. In the present paper, statistics are collected in time (as opposed to ensemble averages) and therefore $\mathcal{A}_{t}$ vanishes.

- $4 \mathcal{A}=\frac{\partial \frac{U_{i}^{+}+U_{i}^{-}}{2}\left\langle\delta q^{2}\right\rangle}{\partial x_{i}}$ is the advection term. This term represents the transport of $\left\langle\delta q^{2}\right\rangle$ in physical space $x_{i}$ by the mean flow. In fact, integrating $\mathcal{A}$ over a volume $\mathcal{V}_{x}$ in physical space and using Gauss' theorem yields $\iiint_{\mathcal{V}_{x}} \mathcal{A d} V=\oiint_{\partial \mathcal{V}_{x}} \frac{U_{i}^{+}+U_{i}^{-}}{2}\left\langle\delta q^{2}\right\rangle n_{i} \mathrm{~d} S$ which is the integral of a flux through $\partial \mathcal{V}_{x}$, the boundary of $\mathcal{V}_{x}$. If this volume encompasses a set of mean streamlines, the flux integral is proportional to the difference of $\left\langle\delta q^{2}\right\rangle$ between the downstream and upstream boundaries in the case of homogeneous turbulence, in which case $\mathcal{A}$ accounts for the decay of $\left\langle\delta q^{2}\right\rangle$ in the direction of the mean flow Hill 2002b; Thiesset et al. 2013a).

- $4 \Pi=\frac{\partial\left\langle\delta u_{i} \delta q^{2}\right\rangle}{\partial r_{i}}$ is the non-linear inter-scale transfer rate and accounts for the effect of non-linear interactions in redistributing $\delta q^{2}$ within the $r_{i}$ space and is given by the divergence in scale space of the flux $\left\langle\delta u_{i} \delta q^{2}\right\rangle$. If one takes the integral of $\Pi$ over a volume in scale space $\mathcal{V}_{r}$, then, similarly to the discussion above, one obtains the flux 
integral $\oiint_{\partial \mathcal{V}_{r}}\left\langle\delta u_{i} \delta q^{2}\right\rangle n_{i} \mathrm{~d} S$. If $\mathcal{V}_{r}$ is taken to be a sphere of radius $\ell$, then $\iiint_{\mathcal{V}_{r}} \Pi \mathrm{d} V$ is proportional to the orientation-averaged flux $\left\langle\delta \mathbf{u} \delta q^{2}\right\rangle$ in scale space, corresponding to a length-scale equal to the radius of the sphere.

- $4 \Pi_{U}=\frac{\partial \delta U_{i}\left\langle\delta q^{2}\right\rangle}{\partial r_{i}}$ is the linear inter-scale transfer rate. Similarly to $\Pi$, this term accounts for transfer of $\delta q^{2}$ in scale space $r_{i}$, but instead of $\delta u_{i}$, it is the two-point difference of mean velocity $\delta U_{i}$ that transports $\delta q^{2}$ in scale space.

- $4 \mathcal{P}=-2\left\langle\delta u_{i} \delta u_{j}\right\rangle \frac{\partial \delta U_{j}}{\partial r_{i}}-\left\langle\left(u_{i}^{+}+u_{i}^{-}\right) \delta u_{j}\right\rangle \frac{\partial \delta U_{j}}{\partial x_{i}}$ can be associated with the production of $\left\langle\delta q^{2}\right\rangle$ by mean flow gradients. Writing $4 \mathcal{P}$ in terms of $x_{i}^{ \pm}=x_{i} \pm r_{i} / 2$ yields $-2\left\langle u_{i}^{+} u_{j}^{+}\right\rangle \frac{\partial U_{i}^{+}}{\partial x_{j}^{+}}-2\left\langle u_{i}^{-} u_{j}^{-}\right\rangle \frac{\partial U_{i}^{-}}{\partial x_{j}^{-}}+2\left\langle u_{i}^{-} u_{j}^{+}\right\rangle \frac{\partial U_{i}^{+}}{\partial x_{j}^{+}}+2\left\langle u_{i}^{+} u_{j}^{-}\right\rangle \frac{\partial U_{i}^{-}}{\partial x_{j}^{-}}$where the first two terms represent the production terms in the one-point turbulent kinetic energy equation and the last two represent combined actions of the mean flow gradients and the two-point correlation tensors $\left\langle u_{i}^{ \pm} u_{j}^{\mp}\right\rangle$ (see e.g. Lindborg 1996, where such terms are related to the equation for $\left.\left\langle\delta u_{i} \delta u_{j}\right\rangle\right)$. Furthermore, if one writes the interscale energy budget for $\delta Q^{2}=\delta U_{i} \delta U_{i}$, which is the mean-flow equivalent to (2.1), the term $\mathcal{P}$ appears as it does in (2.1) but with the opposite sign:

$$
\begin{aligned}
& \frac{\partial \delta Q^{2}}{\partial t}+ \frac{\partial \frac{U_{i}^{+}+U_{i}^{-}}{2} \delta Q^{2}}{\partial x_{i}}+\frac{\partial\left\langle\delta u_{i} \delta \mathbf{u} \cdot \delta \mathbf{U}\right\rangle}{\partial r_{i}}+\frac{\partial \delta U_{i} \delta Q^{2}}{\partial r_{i}}=2\left\langle\delta u_{i} \delta u_{j}\right\rangle \frac{\partial \delta U_{j}}{\partial r_{i}}+ \\
&+\left\langle\left(u_{i}^{+}+u_{i}^{-}\right) \delta u_{j}\right\rangle \frac{\partial \delta U_{j}}{\partial x_{i}}-\frac{\partial\left\langle\frac{u_{i}^{+}+u_{i}^{-}}{2} \delta \mathbf{u} \cdot \delta \mathbf{U}\right\rangle}{\partial x_{i}}-2 \frac{\partial\left\langle\delta U_{i} \delta P\right\rangle}{\partial x_{i}}+\nu \frac{1}{2} \frac{\partial^{2} \delta Q^{2}}{\partial x_{i} \partial x_{i}}+ \\
&+2 \nu \frac{\partial^{2} \delta Q^{2}}{\partial r_{i} \partial r_{i}}-4 \nu\left(\left\langle\frac{\partial \delta U_{j}}{\partial x_{i}} \frac{\partial \delta U_{j}}{\partial x_{i}}\right\rangle+\frac{1}{4}\left\langle\frac{\partial \delta U_{j}}{\partial r_{i}} \frac{\partial \delta U_{j}}{\partial r_{i}}\right\rangle\right)
\end{aligned}
$$

It follows that the interscale energy budget for $\left\langle\left[\left(U_{i}^{+}+u_{i}^{+}\right)-\left(U_{i}^{-}+u_{i}^{-}\right)\right]\left[\left(U_{i}^{+}+u_{i}^{+}\right)-\left(U_{i}^{-}+\right.\right.\right.$ $\left.\left.\left.u_{i}^{-}\right)\right]\right\rangle$, which equals $\delta Q^{2}+\left\langle\delta q^{2}\right\rangle$, does not involve $\mathcal{P}$. This observation consolidates the interpretation of $\mathcal{P}$ as a production term. When $\mathcal{P}$ is positive/negative, energy is therefore lost/gained by $\delta Q^{2}$ and gained/lost by $\left\langle\delta q^{2}\right\rangle$ at the same rate. This process is only significant at large enough values of $r$ where $\delta Q^{2}$ is not negligible. Note that $\frac{\partial\left\langle\delta u_{i} \delta \mathbf{u} \cdot \delta \mathbf{U}\right\rangle}{\partial r_{i}}$ is the difference between the interscale energy transfer rate of the total two-point kinetic energy, $\frac{\partial\left\langle\delta u_{i} \frac{1}{2}(\delta \mathbf{U}+\delta \mathbf{u})^{2}\right\rangle}{\partial r_{i}}$, and the interscale energy transfer rate $2 \Pi=\frac{\partial\left\langle\delta u_{i} \delta q^{2}\right\rangle}{\partial r_{i}}$ of $\frac{1}{2} \delta q^{2}$. All the other terms in (2.3) can be interpreted in the same way as their analogues in (2.1), except for the term $\frac{\partial\left\langle\frac{u_{i}^{+}+u_{i}^{-}}{2} \delta \mathbf{u} \cdot \delta \mathbf{U}\right\rangle}{\partial x_{i}}$ which we discuss in the next bullet point.

- $4 \mathcal{T}_{u}=-\frac{\partial\left\langle\frac{u_{i}^{+}+u_{i}^{-}}{2} \delta q^{2}\right\rangle}{\partial x_{i}}$ is the transport of $\delta q^{2}$ in physical space due to turbulent fluctuations. It features in (2.1) whereas the term $\frac{\partial\left\langle\frac{u_{i}^{+}+u_{i}^{-}}{2} \delta \mathbf{u} \cdot \delta \mathbf{U}\right\rangle}{\partial x_{i}}$ which features in (2.3) is the difference between the turbulent transport of total two-point kinetic energy $\frac{1}{2}(\delta \mathbf{U}+$ $\delta \mathbf{u})^{2}$ in physical space and $2 \mathcal{T}_{u}$.

- $4 \mathcal{T}_{p}=-2 \frac{\partial\left\langle\delta u_{i} \delta p\right\rangle}{\partial x_{i}}$ is equal to -2 times the correlation between velocity differences and differences of pressure gradient.

- $4 \mathcal{D}_{x}=\nu \frac{1}{2} \frac{\partial^{2}\left\langle\delta q^{2}\right\rangle}{\partial x_{i} \partial x_{i}}$ is the diffusion in physical space due to viscosity. This term is analogous to the diffusion term appearing in the single-point turbulent kinetic energy equation and thus it's contribution to (2.1) is expected to be small.

- $4 \mathcal{D}_{r}=2 \nu \frac{\partial^{2}\left\langle\delta q^{2}\right\rangle}{\partial r_{i} \partial r_{i}}$ is the diffusion in scale space by viscosity. This term is equal to the dissipation $\varepsilon$ when the two points coincide $(r=0)$ and can be shown (see Appendix B in 
Valente \& Vassilicos 2015) to be negligible for separations much larger than the Taylor micro-scale.

- $\varepsilon_{r}$ is the two-point average dissipation rate $\varepsilon_{r}=\frac{\varepsilon^{+}+\varepsilon^{-}}{2}$ since it equals $\frac{1}{2} \nu\left(\left\langle\frac{\partial u_{j}^{+}}{\partial x_{i}^{+}} \frac{\partial u_{j}^{+}}{\partial x_{i}^{+}}\right\rangle+\left\langle\frac{\partial u_{j}^{-}}{\partial x_{i}^{-}} \frac{\partial u_{j}^{-}}{\partial x_{i}^{-}}\right\rangle\right)$.

If a turbulent flow is locally homogeneous over length-scales $r$ smaller than a certain inhomogeneity length-scale, then (2.3) reduces to the identity $0=0$ and (2.1) reduces to

$$
\mathcal{A}_{t}+\mathcal{A}+\Pi \approx \mathcal{D}_{r}-\varepsilon
$$

for such length-scales $r$. Given that $\mathcal{D}_{r}$ is negligible at length-scales $r$ larger than the local Taylor length-scale, (see Appendix B of Valente \& Vassilicos 2015), one is left with

$$
\mathcal{A}_{t}+\mathcal{A}+\Pi \approx-\varepsilon
$$

in the intermediate range of $r$ between the Taylor length-scale and the inhomogeneity length-scale (assuming we are considering a region of a turbulent flow where such a range exists). Kolmogorov's hypothesis of local equilibrium then leads to the equilibrium relation

$$
\Pi \approx-\varepsilon
$$

which is the central property of the Kolmogorov equilibrium cascade in locally homogeneous turbulence. A further step is needed to obtain Kolmogorov's $\left\langle\delta q^{2}>\sim \varepsilon^{2 / 3} r^{2 / 3}\right.$ and $<\delta u_{i}^{2}>\sim \varepsilon^{2 / 3} r_{i}^{2 / 3}$ for $i=1,2,3$ and the Kolmogorov-Obukhov $-5 / 3$ energy spectrum (Obukhov 1941). This step requires additional hypotheses of small-scale isotropy and self-similarity, and proceeds either by dimensional analysis Kolmogorov (1941a) or by taking the skewness of the velocity differences to be constant Kolmogorov (1941c).

Due to experimental limitations which prevent explicit measurement and computation of many terms in (2.1), the laboratory studies of Thiesset et al. (2011a), Thiesset et al. $(2011 b)$, Thiesset et al. (2013a), Thiesset et al. (2014), Valente \& Vassilicos (2015) and Hearst \& Lavoie (2014) have focused on (2.4), mostly in an intermediate region of planar wakes (at streamwise distances larger than $10 d$ and typically $40 d$ from the wake generator of size $d$ ). Gomes-Fernandes et al. (2015) attempted to measure and compute as many of the terms in (2.1) as possible from a planar two-component PIV in the very near field of a turbulence generated by a fractal square grid. Their results suggest that, upstream of the peak of turbulent kinetic energy where the turbulence is very inhomogeneous and anisotropic and therefore not decaying but rather building up, the inter-scale transfer rate $\Pi$ is approximately constant within a sizeable range of scales. A constant $\Pi$ over a range of scales is what (2.6) would predict for a locally homogeneous turbulence. However, the measurements of Gomes-Fernandes et al. (2015) suggested that all the terms appearing in (2.1) have a non-negligible contribution, the exact opposite of what the Kolmogorov theory assumes for the purpose of deriving (2.6). Furthermore, Gomes-Fernandes et al. (2015) observed a combined forward and inverse cascade in scale space in the region of their measurements. Specifically their results suggested that the interscale energy flux $\left\langle\delta u_{i} \delta q^{2}\right\rangle \frac{r_{i}}{r}$ has different signs at different orientations. However, some of the terms in eq. (2.1) were inaccessible to Gomes-Fernandes et al. (2015) due to experimental limitations and others required assumptions to be computed from their data. In this paper we present DNS evidence which confirms and strengthens the conclusions of Gomes-Fernandes et al. (2015) and which permits us to go further and obtain new insights about (2.1) in the near wake of a square prism. 


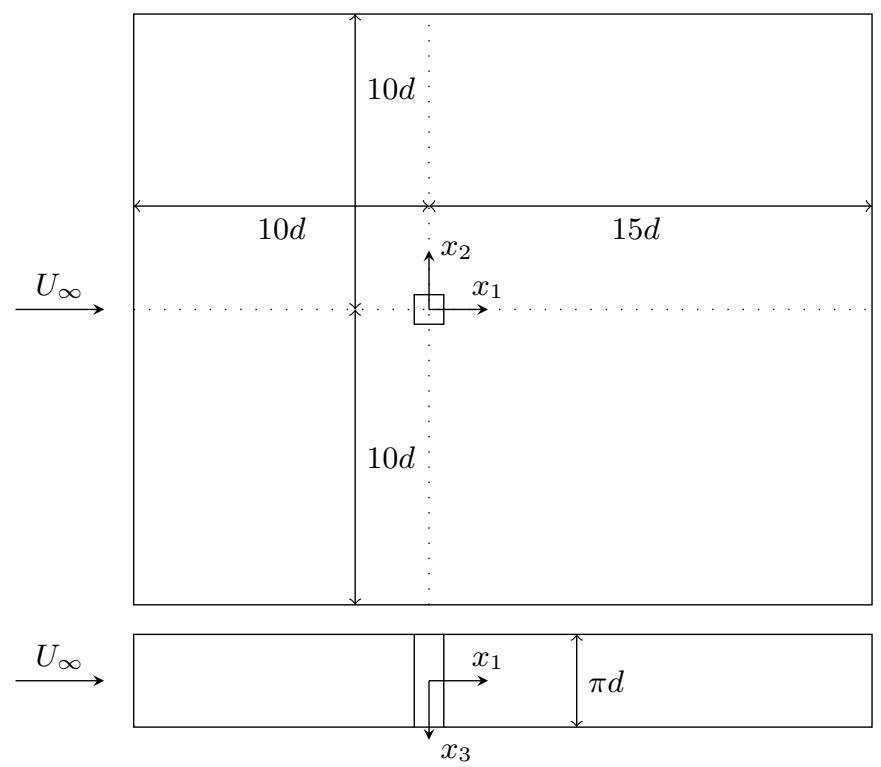

Figure 2: Domain dimensions. Top: side view; bottom: top view. The origin of our coordinate system is at the centre of the square prism.

\subsection{Numerical Method}

For the numerical simulations a cell-centred fully unstructured finite volume (FV) code called Panta Rhei was employed using the PETSc library (Balay et al. 2016) for the algebraic solvers. The coupling between pressure and velocity is achieved via the PISO algorithm (Issa 1986). The time integration is done using a second order backward method while the spatial central discretisation is of second order. The FV approach allows the mesh to be stretched so as to better refine the grid in regions of the flow where small scale dynamics are relevant, such as the core of the wake and the separating shear layer. In section 2.3 we show a comparison with experiments and other simulations of mean flow profiles and integral properties of flow.

The fluid domain is sketched in fig. 2 along with it's dimensions and the reference frame. At the inlet the velocity was set to $U_{\infty}$ and at the outlet, the one dimensional advection equation is solved (convective boundary condition). The spanwise boundaries are treated as periodic while at the top and bottom the Dirichlet condition for pressure and Neumann condition for the velocity reduce blockage effects allowing the flow to be entrained across those boundaries.

The computational grid used had just under 40 million cells. The length of each side of the square prism in the $\left(x_{1}, x_{2}\right)$ plane being $d$, the smallest cell size was $0.0015 d$ and was at the corners of the prism. Over each edge 121 nodes were placed with most nodes being concentrated close to the corners. Near the inlet and top/bottom boundaries the cell sizes were set to $0.325 d$ while near the outlet the cell size was $0.065 d$. Appropriate care was placed in connecting the different grid sizes using stretching functions which allowed for a smooth variation of the cells' dimensions. The mesh was extruded in the spanwise direction generating 150 layers. In order to keep the computational requirements as low as possible, the mesh was stretched (in the $x_{1}$ and $x_{2}$ directions) allowing the finer cells to be located in the area around the prism. This ensured appropriate resolution of the thin separating shear layer. In the core of the wake the resolution was found to be at worse 


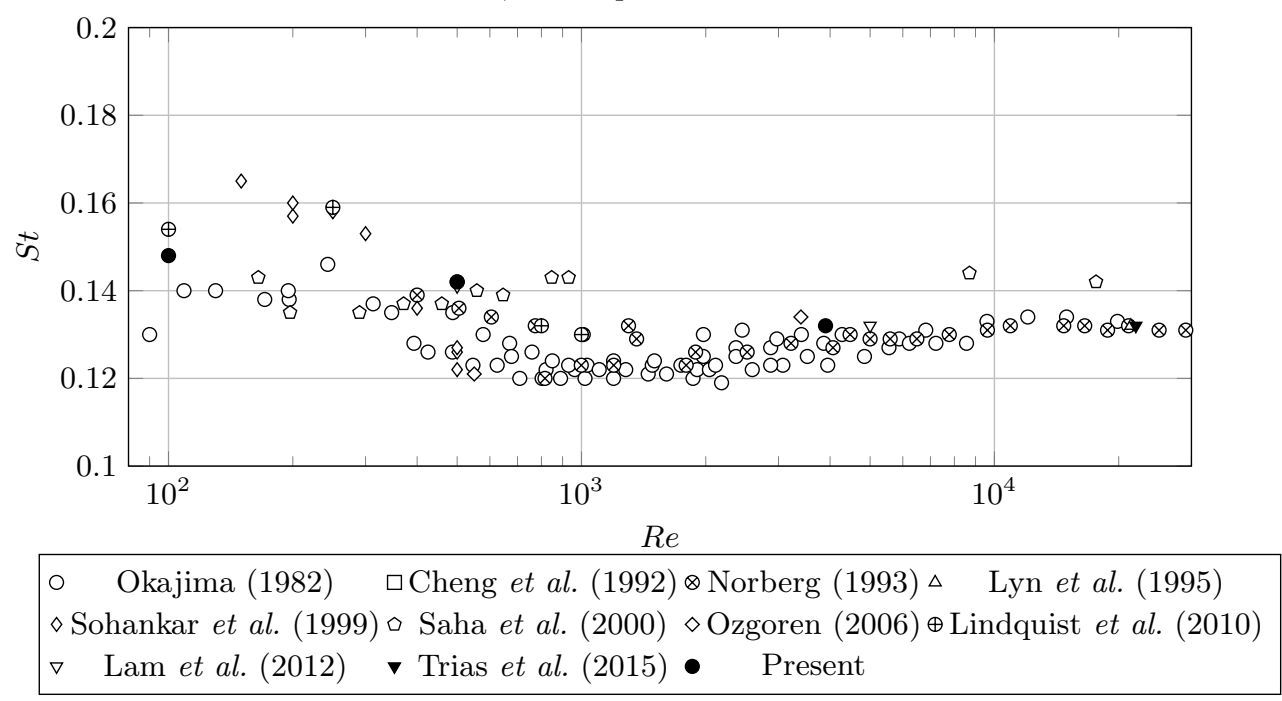

Figure 3: $S t$ versus Re from both experiments and DNS. Repeated symbols (for the same $R e)$ indicate different experimental/numerical conditions.

about 4 times the Kolmogorov length scale $\eta$, in fact, along the centreline the resolution varied between $3.8 \eta$ and $2.9 \eta$ at $x_{1} / d=2$ and $x_{1} / d=8$, respectively.

It was found that a maximum value of the $C F L=u \frac{\Delta t}{\Delta x}$ of about 4 allowed the numerical solution to be stable and that led to an average of 0.2 across the whole domain. The largest values of the $C F L$ were found in areas where the flow was still laminar. The resulting time step was $0.0025 \frac{U_{\infty}}{d}$ which corresponds to about 3000 time steps per shedding cycle. In the core of the wake, this value of the time step is smaller by at least one order of magnitude compared to the Kolmogorov time scale.

The numerical computation of all the terms in (2.1) was done by re-writing them as correlations of velocities, pressure and their derivatives between locations $x_{i}^{+}$and $x_{i}^{-}$ where the FV approach was used to compute the derivatives.

\subsection{Comparison of Mean Flow and Turbulence Intensity Profiles with Literature}

In order to validate our results, we have compared several statistics with those reported in literature. In the present section we discuss statistics of the velocity, as well as the shedding frequency associated with the vortex shedding. In appendix A further comparisons are made for other quantities, such as force coefficients.

In fig. 3 we report values of the normalised shedding frequency, the Strouhal number $S t=\frac{f_{s} d}{U_{\infty}}$ (where $f_{s}$ is the shedding frequency), for three values of $R e(100,500$ and $3900)$. In particular for the $R e=3900$ case, the value of $S t$ was found to be 0.13 . Only the $R e=3900$ simulation is used in the remainder of this paper with the exception of Appendix A where the $R e=100$ and 500 simulations are used for validation purposes.

In figs. 4 to 7 we show the variation along the centreline of $U_{1}, u_{1}^{\prime}, u_{2}^{\prime}$ and $u_{3}^{\prime}$ which are the mean stream-wise velocity and the standard deviations of the stream-wise, crossstream and spanwise velocity components, respectively. Apart from the DNS results of Arslan et al. (2012) and Trias et al. (2015) all remaining references refer to experimental results. The DNS of Arslan et al. (2012) employed a code developed for LES simulations, moreover, they report a resolution in terms of Kolmogorov length scales of about $7 \eta$. The DNS of Trias et al. (2015) on the other hand report a resolution of about $4 \eta$ and even 


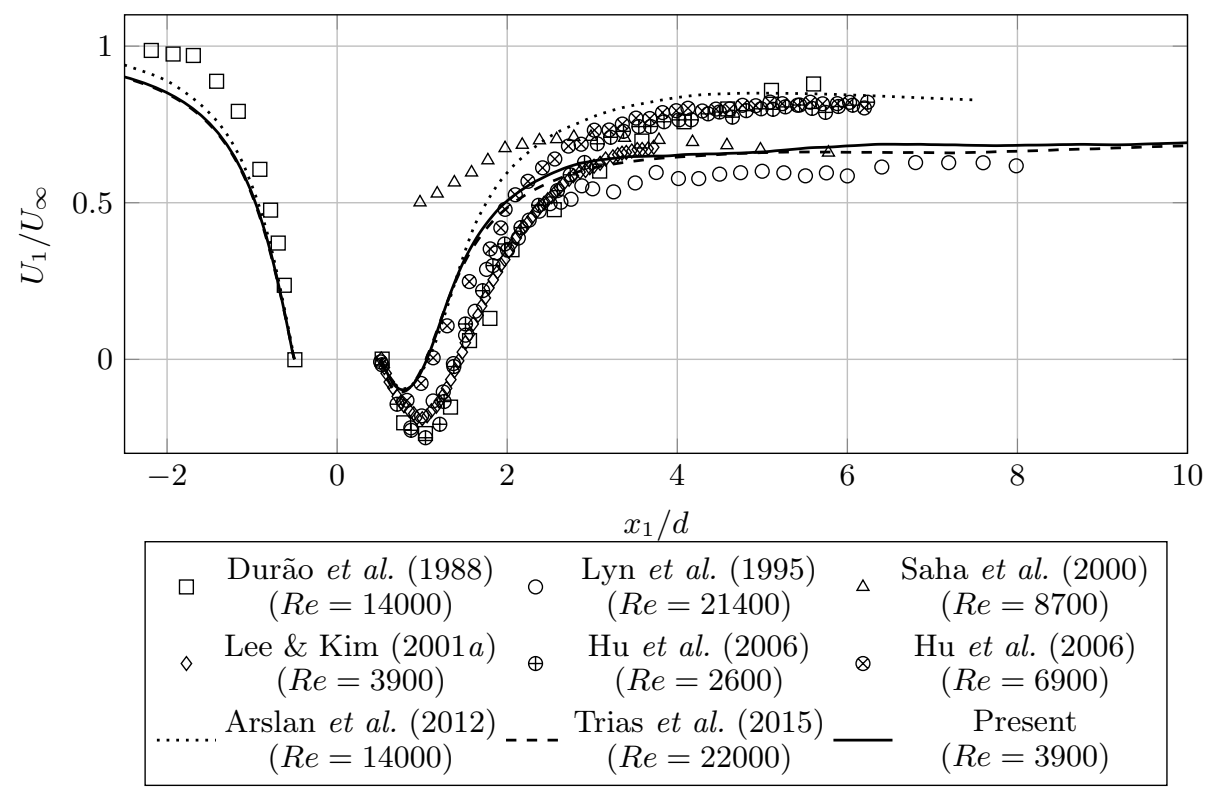

Figure 4: Profile of $U_{1}$ normalised by $U_{\infty}$ along the geometrical centreline for $-2.5<$ $x_{1} / d<10$. Experimental results from different references are indicated by symbols while the lines indicate numerical (DNS) results.

though they simulate a much larger $R e$ flow, we find a very good agreement between our stream-wise profiles and theirs (database available in ref. [36] of their paper).

Significant scatter in the statistics of the velocity is observed when comparing different references. In particular, the estimation of the maximum recirculation velocity and rearstagnation point appears to be drastically different between experiments and DNS. This is in line with the results reported in Voke (1996) and Sohankar (2006) where LES simulations at $R e=22000$ are compared with the reference data of Lyn et al. (1995). On the other hand, significant differences are also observed in the recovery velocity: while the present results and those of Lyn et al. $(\underline{1995})$, Lee \& Kim (2001b) and Trias et al. $(\underline{2015})$ show a very slow increase of $U_{1} / U_{\infty}$ along $x_{1} / d$ from the value of about 0.6 at $x_{1} / d \approx 3$ to around 0.7 at $x_{1} / d \approx 8$ others find the mean velocity to be over $20 \%$ higher. It is unlikely that these differences are solely due to wind tunnel blockage and free-stream turbulence. Lyn et al. (1995), Lee \& Kim (2001b) and Arslan et al. (2012) reported a blockage of $7 \%$ but $\mathrm{Hu}$ et al. (2006) and Trias et al. (2015) report a blockage of about $2 \%$ even though there are differences in their results. The free-stream turbulence on the other hand appears to have a more significant effect on $U_{1} / U_{\infty}$ in the experiment of Durão et al. (1988) who report $6 \%$ free-stream turbulence; this explains the differences observed in the incoming profiles.

As seen in figs. 5 to 7 the disparity between the different references in the statistics of fluctuating velocities are even more significant than for $U_{1} / U_{\infty}$, particularly in the very near wake. The large values of $u_{1}^{\prime}$ observed at $x_{1} / d<-0.5$ in the data of Durão et al. (1988) are due to their fairly high free-stream turbulence of $6 \%$. While all references agree that the peak of $u_{1}^{\prime}$ should occur just after $x_{1} \approx d$, the actual value of $u_{1}^{\prime} / U_{\infty}$ at its peak varies between 0.28 and 0.56 . We find the peak value of $u_{1}^{\prime} / U_{\infty}$ to be close to 0.41 which is in agreement with the results of Lyn et al. (1995) and Trias et al. (2015) which were obtained at a larger $R e$. 


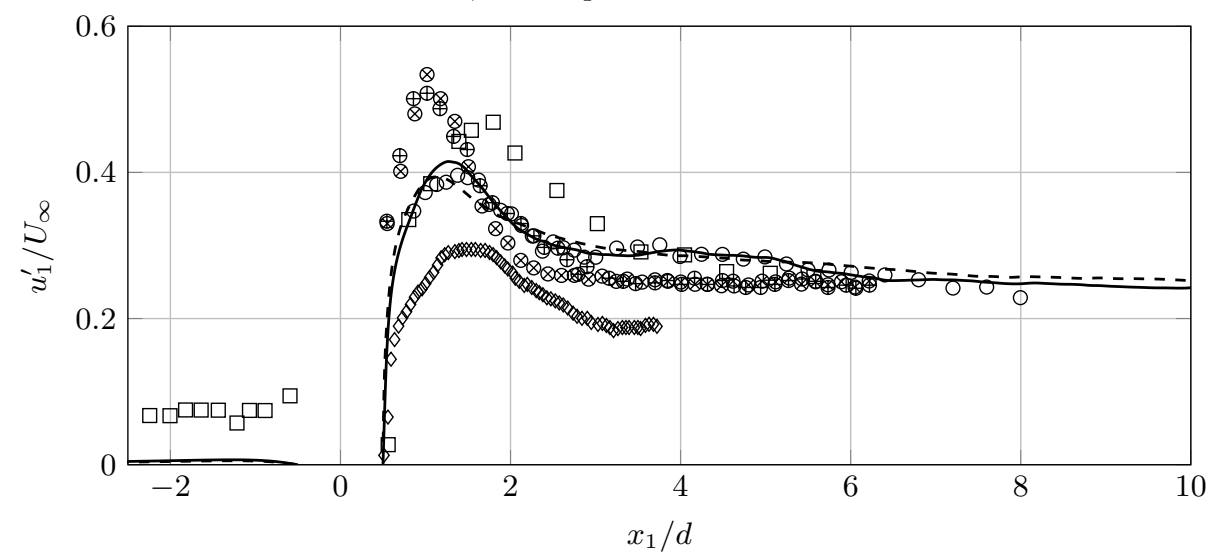

Figure 5: Profile of $u_{1}^{\prime}=\sqrt{\left\langle u_{1}^{2}\right\rangle}$ normalised by $U_{\infty}$ along the geometrical centreline for $-2.5<x_{1} / d<10$. Same legend as in fig. 4. Turbulent statistics of Lee \& Kim (2001a) are shown in Lee \& Kim (2001b).

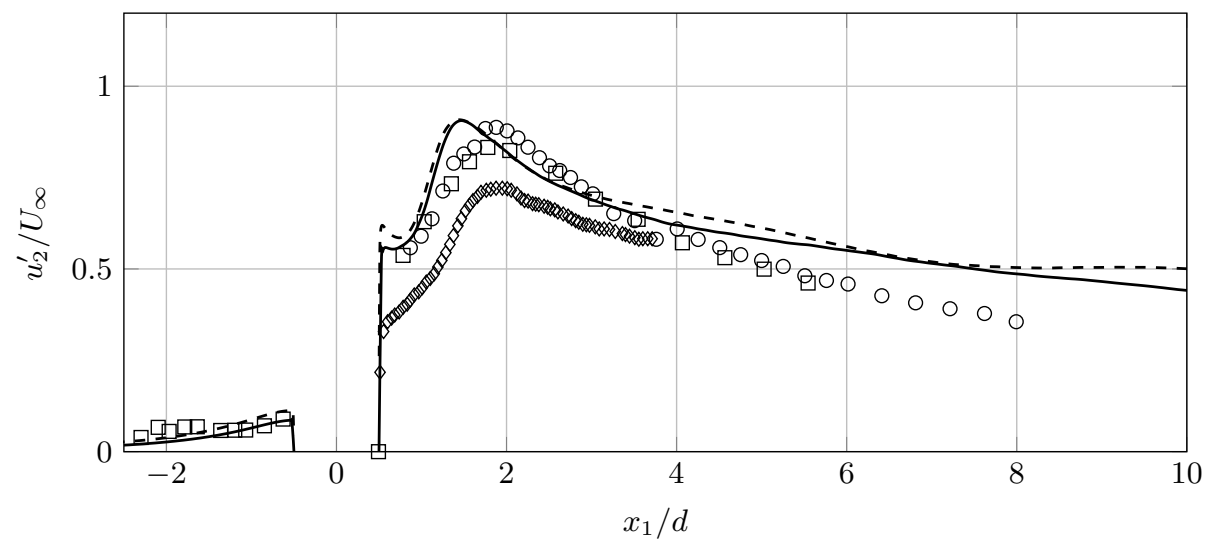

Figure 6: Profile of $u_{2}^{\prime}=\sqrt{\left\langle u_{1}^{2}\right\rangle}$ normalised by $U_{\infty}$ along the geometrical centreline for $-2.5<x_{1} / d<10$. Same legend as in fig. 4. Turbulent statistics of Lee \& Kim (2001a) are shown in Lee \& Kim (2001b).

The intensity of cross-stream fluctuations is mostly determined by the shedding. In fact, the distance from the prism to the location of the peak of $u_{2}^{\prime} / U_{\infty}$ is used to define the vortex formation length. Both the present results and those of Trias et al. $(2015)$ show a vortex formation length extending to $x_{1} / d \approx 1.5$, while the experiments of Durão et al. (1988), Lyn et al. (1995) and Lee \& Kim $(2001 b)$ place this further downstream, at $x_{1} / d \approx 1.9$. Apart from the PIV results of Lee \& Kim (2001b), the peak value of $u_{2}^{\prime} / U_{\infty}$ is reported to be close to 0.9 both in the present results and Lyn et al. (1995) and Trias et al. (2015) and just over 0.8 in Durão et al. (1988). Differences are also observed in the decay of $u_{2}^{\prime} / U_{\infty}$ with increasing stream-wise distance. Although Lee \& Kim (2001b) did not measure sufficiently downstream, their results seem to agree with the present DNS and that of Trias et al. (2015) in a slower decrease of $u_{2}^{\prime} / U_{\infty}$ with $x_{1} / d$ in comparison to Durão et al. (1988) and Lvn et al. (1995).

Fewer results are available for the spanwise fluctuating velocity. The hot-wire measurements of $\mathrm{Hu}$ et al. (2006) at two different Re show different evolutions of $u_{3}^{\prime} / U_{\infty}$ 


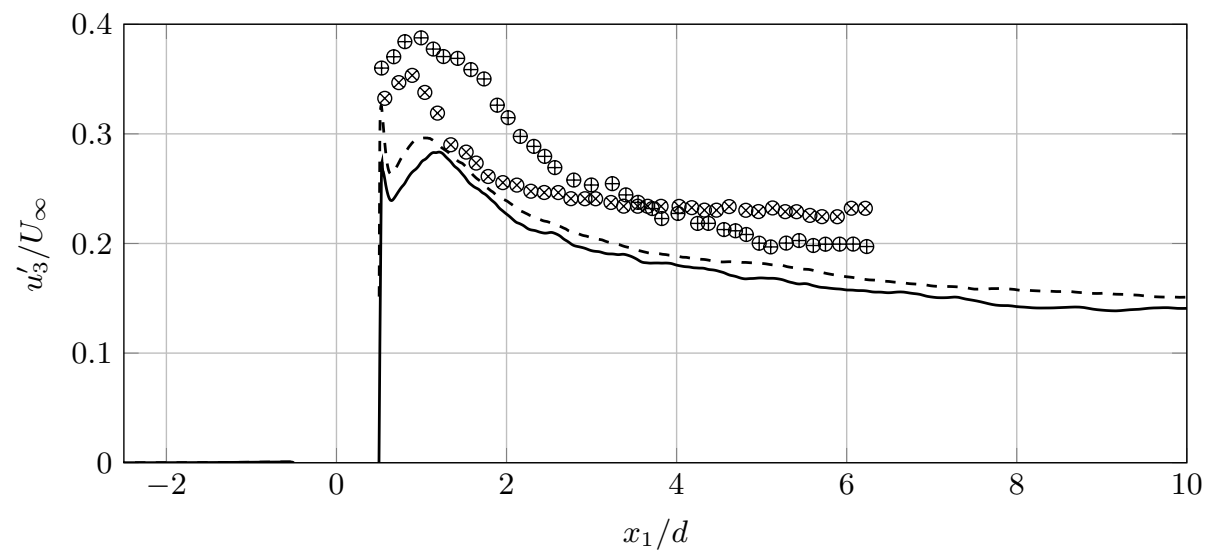

Figure 7: Profile of $u_{3}^{\prime}=\sqrt{\left\langle u_{3}^{2}\right\rangle}$ normalised by $U_{\infty}$ along the geometrical centreline for $-2.5<x_{1} / d<10$. Same legend as in fig. 4 .

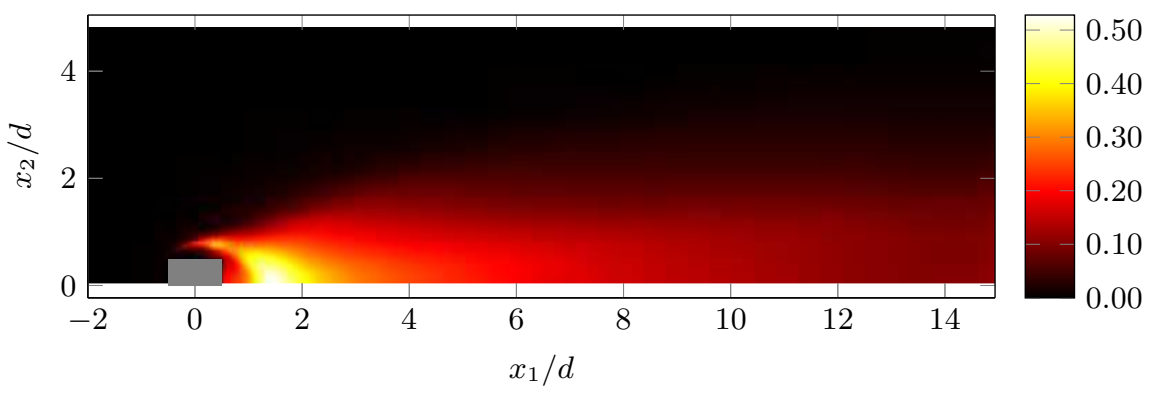

Figure 8: Contour of mean turbulent kinetic energy normalised by $U_{\infty}^{2}$.

with $x_{1} / d$, while the present DNS at $R e=3900$ shows the same profile of $u_{3}^{\prime} / U_{\infty}$ as in Trias et al. (2015) whose results refer to $R e=22000$. In the DNS, the peak of $u_{3}^{\prime} / U_{\infty}$ on the centreline appears to be at the same location as the mean stagnation point (where $\left.U_{1}=0\right)$.

Finally, we show in fig. 8 the contour of the normalised turbulent kinetic energy $\frac{u_{1}^{\prime 2}+u_{2}^{\prime 2}+u_{3}^{\prime 2}}{2} / U_{\infty}^{2}$. The peak turbulent kinetic energy occurs at the centreline, due to the large contribution of $u_{2}^{\prime}$ which also peaks at the centreline. However, it is possible to see another peak (of smaller magnitude) roughly at the stagnation point in between the two large recirculation bubbles at the top/bottom and back (seefig. 37 in appendix A). This is most likely due to the flapping of the shear layer as it corresponds also to a peak in $u_{1}^{\prime} / U_{\infty}$.

The convergence of the one-point statistics in this section and of two-point statistics such as those in the following sections is presented in appendix B.

\section{Spectra and Structure Functions}

\subsection{Energy Spectra in Frequency Domain and Taylor's Hypothesis}

We start by looking at turbulence spectra in the region of the flow where we study the scale-by-scale energy budget (2.1) in the next section. The energy spectra at several locations along the centreline are shown in figs. 9a, 10a and 10b for the stream-wise, cross-stream and spanwise components of the fluctuating velocity, respectively. At these 

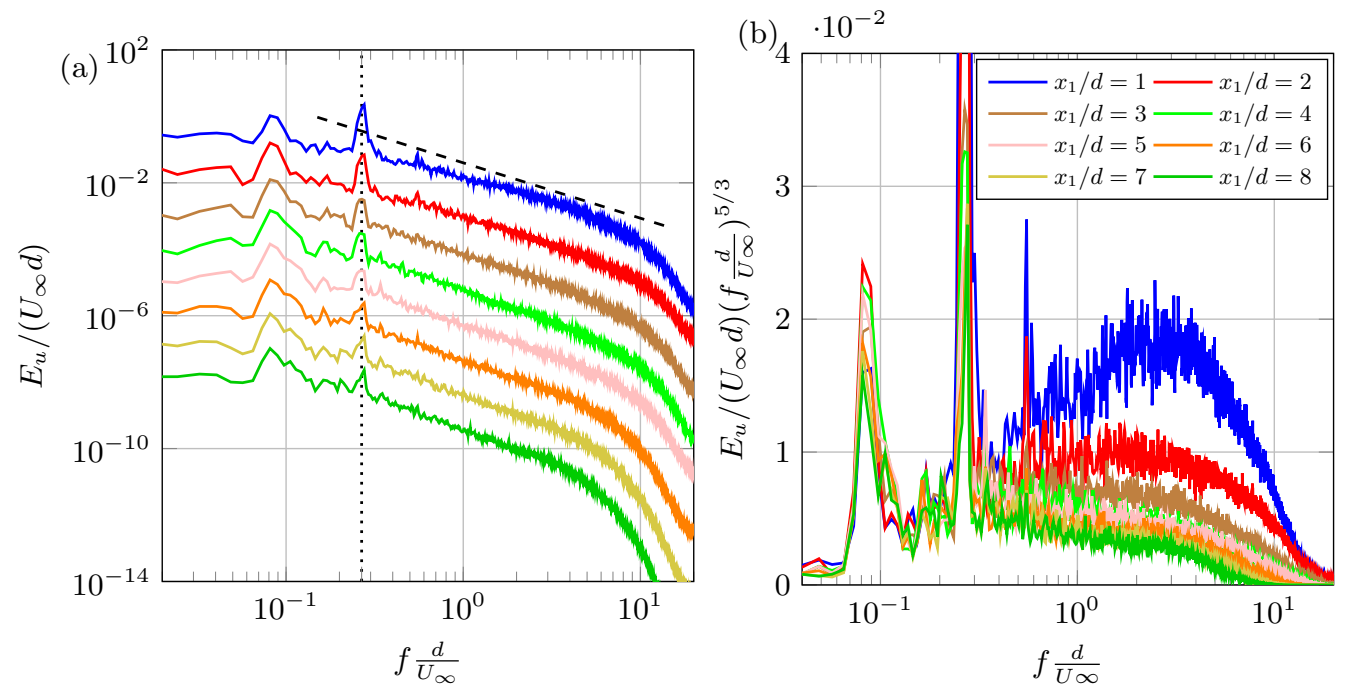

Figure 9: Power spectrum densities of stream-wise fluctuating velocity at eight locations on the centreline (different locations are offset by one decade) normalised by $U_{\infty} d$. In (a) the dashed line indicates a slope of $-5 / 3$ and the dotted line indicates $f=2 f_{s}$; in (b) compensated spectra are plotted in linear-logarithmic axes.

locations, the spectra display a power law which is close to $-5 / 3$ over a substantial range of frequencies at $x_{1} / d=2$. As the distance $x_{1}$ to the prism increases, this power law deteriorates while the range of frequencies narrows. This is in spite of the flow becoming more homogeneous and undergoing an increase in local Reynolds' number. In fig. $9 \mathrm{~b}$ the $5 / 3$ compensated spectra of the stream-wise fluctuations are shown in a linear-logarithmic plot to highlight that the spectra are close to a $-5 / 3$ power law over just under a decade of frequencies, particularly at $x_{1}=2 d$ which is located straight after the peak of turbulent kinetic energy (as seen in fig. 8). As will become fully clear in the following section, this observation is in-line with the comment made by Kraichnan (1974) that such a power law is observed in regions of flows where Kolmogorov's theory is not expected to hold. Several other authors have reported such a power law in simulations and experiments of planar wakes (see e.g. Cantwell \& Coles 1983; Ong \& Wallace 1996; Ma et al. 2000; Kravchenko \& Moin 2000; Braza et al. 2006; Wissink \& Rodi 2008; Lehmkuhl et al. 2013; Trias et al. 2015) at comparable distances from the wake generator (on the centreline) and similar Reynolds' numbers.

Less reported by previous authors, however, is the fact that the range of frequencies over which a power law is observed appears to decrease with increasing downstream distance in agreement with a similar observation made by Laizet et al. (2015) in near-field grid-generated turbulence. It is also worth mentioning that the local Taylor length-based Reynolds number $R e_{\lambda}=\frac{u_{1}^{\prime} \lambda}{\nu}$, where $\lambda=\sqrt{15 \frac{\nu}{\varepsilon}} u_{1}^{\prime}$, increases from about 120 at $x_{1} / d=2$ to about 170 at $x_{1} / d=10$. This is a first indication that these near $-5 / 3$ power laws do not have much to do with Kolmogorov theory where an increase in Reynolds number is expected to lead to a broader inertial range.

Due to the predominantly planar nature of the vortex shedding only the spectra of the stream-wise and cross-stream components display pronounced peaks (figs. 9a and 10a) while this is not the case for the spectra of spanwise fluctuations (fig. 10b). For the stream-wise component, the peak appears at $2 f_{s}$ while for the cross-stream component 


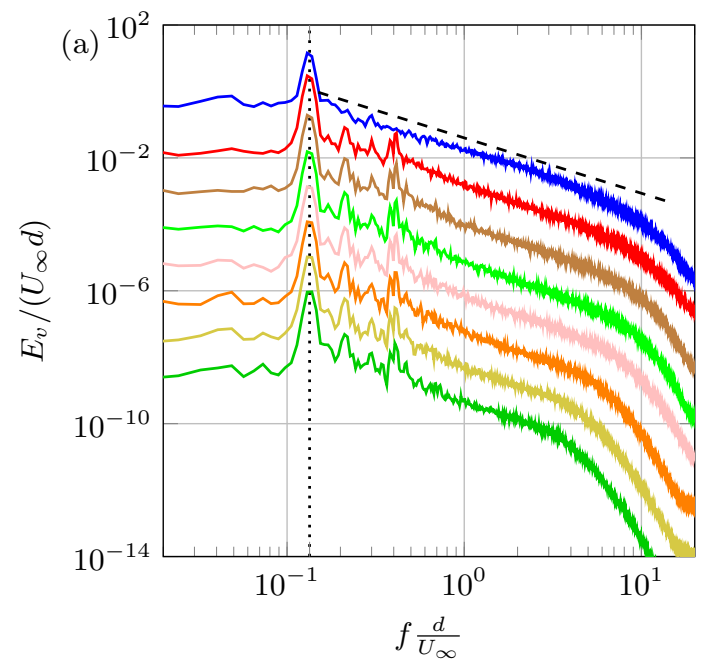

(b)

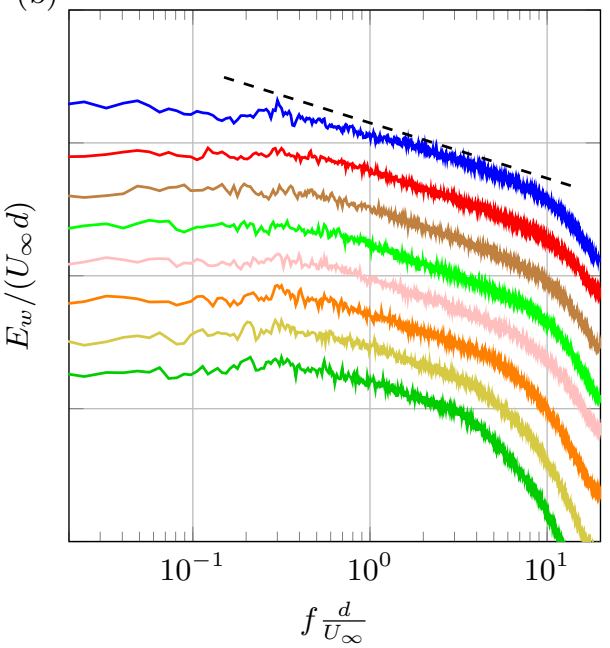

Figure 10: Power spectrum densities, normalised by $U_{\infty} d$, of the cross-stream and spanwise velocity components (a) and (b), respectively (different locations are offset by one decade). See fig. 9 for legend. The dashed line indicates a slope of $-5 / 3$. In (a) the dotted line indicates $f=f_{s}$.

the peak occurs at $f_{s}$. This is due to the alternating vortices shed from the top and bottom sides having spanwise vorticity of opposite signs, thus inducing on the centreline stream-wise fluctuations in the same direction but cross stream fluctuations in opposite directions.

The $-5 / 3$ power law spectrum obtained by the Kolmogorov theory is for wavenumber spectra (Obukhov 1941), not frequency spectra. The conversion from wavenumber to frequency required to interpret spectra obtained from velocity data collected over time at a particular point in space is usually based on Taylor's frozen turbulence hypothesis (introduced in Taylor 1935). According to this hypothesis, high enough frequencies $f$ are related to high enough wavenumbers $k$ by $k=2 \pi f / U_{c}$ where $U_{c}$ is a convection velocity $U_{c}$, usually the mean flow velocity at the data collection point. Given that we find well-defined power laws in our frequency spectra with exponents more or less close to $-5 / 3$ it makes sense to test the validity of Taylor's hypothesis at those points where these frequency spectra were found.

To verify Taylor's hypothesis, we follow the approach of Laizet et al. (2015) and compute the correlation coefficient

$$
\rho_{u}\left(x_{1}, \Delta x, \Delta t\right)=\frac{\left\langle u_{1}\left(x_{1}, t\right) u_{1}\left(x_{1}+\Delta x, t+\Delta t\right)\right\rangle}{\sqrt{\left\langle u_{1}\left(x_{1}, t\right)^{2}\right\rangle\left\langle u_{1}\left(x_{1}+\Delta x, t+\Delta t\right)^{2}\right\rangle}}
$$

where the velocity component $u_{1}$ is of course also function of $x_{2}$ and $x_{3}$ in principle but the correlation is evaluated at $x_{2}=x_{3}=0$. Infig. 11a, $\rho_{u}$ is plotted for $x_{1} / d=2$ and for five different values of the spatial offset $\Delta x$. Even at separations as large as $\Delta x / d=1$ there is a strong correlation of the velocity signals. The peaks of the correlation $\rho_{u}$ can be used to compute $U_{c}$ by taking the ratio between the values of $\Delta x$ and $\Delta t$ where these peaks occur.

As shown in figs. 11b and 11c, there does seem to be a linear relationship between $\Delta x$ and $\Delta t$ suggesting that $U_{c}$ is well-defined, which supports the validity of the Taylor hypothesis. As is also clear in figs. 11b and 11c $U_{c}$ is slightly larger than the mean 

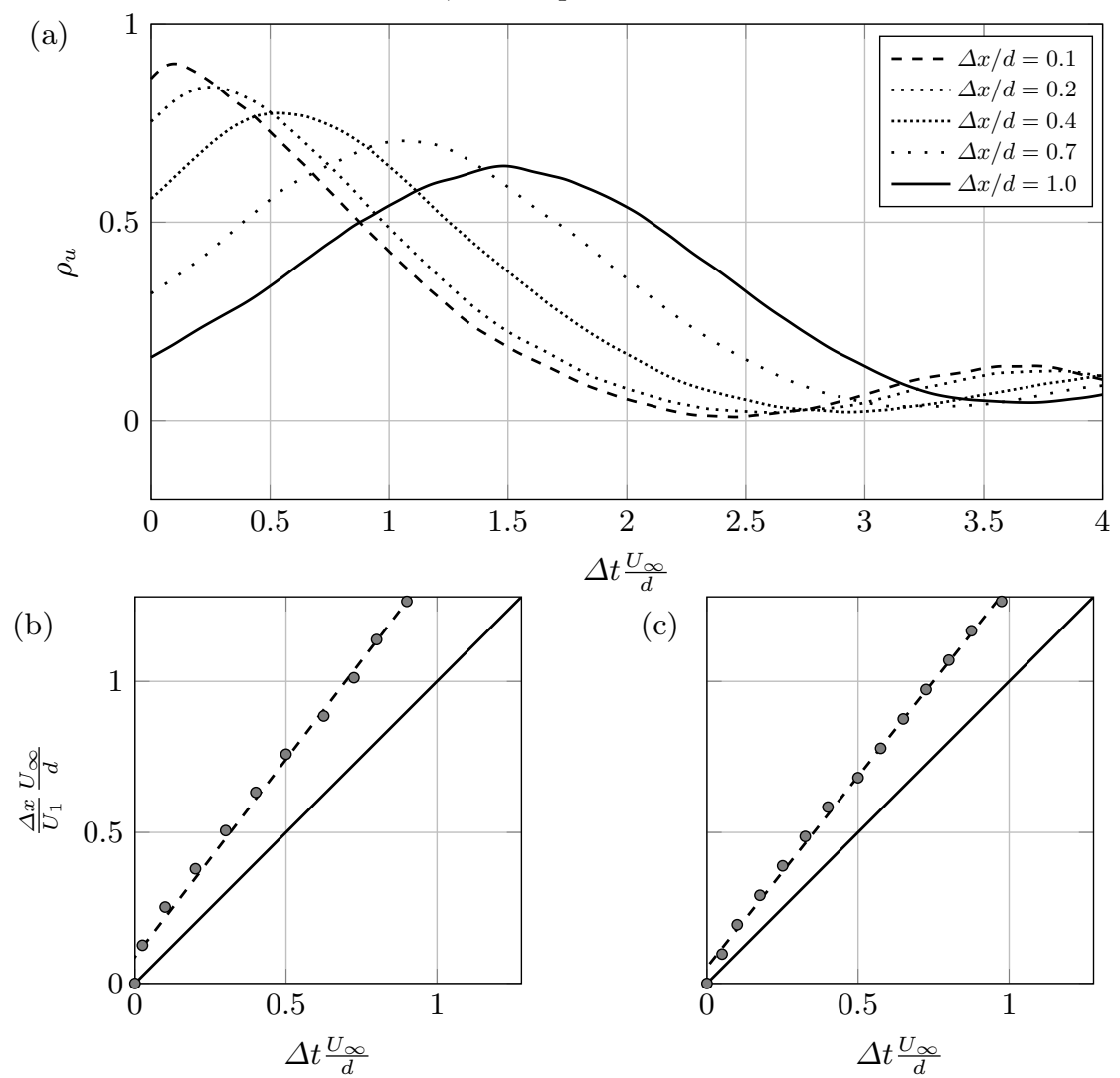

Figure 11: In (a) the correlation coefficient eq. (3.1) at $x_{1} / d=2$ on the centreline is plotted for different separations $\Delta x$. In (b) and (c) the symbols indicate the locations of the peaks of eq. (3.1) at both $x_{1} / d=2$ (b) and $x_{1} / d=4$ (c) on the centreline, the full lines indicate the slope $U_{c} / U_{1}=1$ and the dashed lines indicate linear fits to the data.

flow velocity on the centreline. For example, we have found the values $U_{c} / U_{\infty} \approx 0.67$ and $U_{c} / U_{\infty} \approx 0.83$ at the two centreline locations $x_{1} / d=2$ and $x_{1} / d=4$ respectively, which, as can be seen in fig. 4, are indeed larger than $U_{1}$ at the same locations. In fact, $U_{c}$ appears to be close to the convection velocity of the large scale vortices as reported by Bloor \& Gerrard (1966) and Zhou \& Antonia (1992). Similar conclusions can be reached for the components $u_{2}$ and $u_{3}$ of the fluctuating velocity in terms of a correlation coefficient defined as in (3.1) but for $u_{2}$ and $u_{3}$ respectively. In fact we find $U_{c}\left(x_{1}, 0,0\right) \approx 1.3 U_{1}\left(x_{1}, 0,0\right)$ for all three fluctuating velocity components in the range $x_{1} / d \geqslant 3$ and $U_{c} / U_{1}$ slightly higher but under or close to 1.4 at $x_{1}=2 d$ on the centreline. While many other considerations regarding the conversion from frequency to wavenumber can arise (see Lumley 1965; Wyngaard \& Clifford 1977), it is beyond this paper to delve into such issues. The point of this limited space-time analysis has simply been to confer a little more substance to our observation of near $-5 / 3$ power law energy spectra by providing some evidence in favour of the Taylor frozen turbulence hypothesis which therefore suggests that the power law frequency spectra that we have observed may be reflections of similar underlying wavenumber spectra. In the following subsection we briefly report on second order structure functions as they are Fourier analogues of wavenumber spectra. 

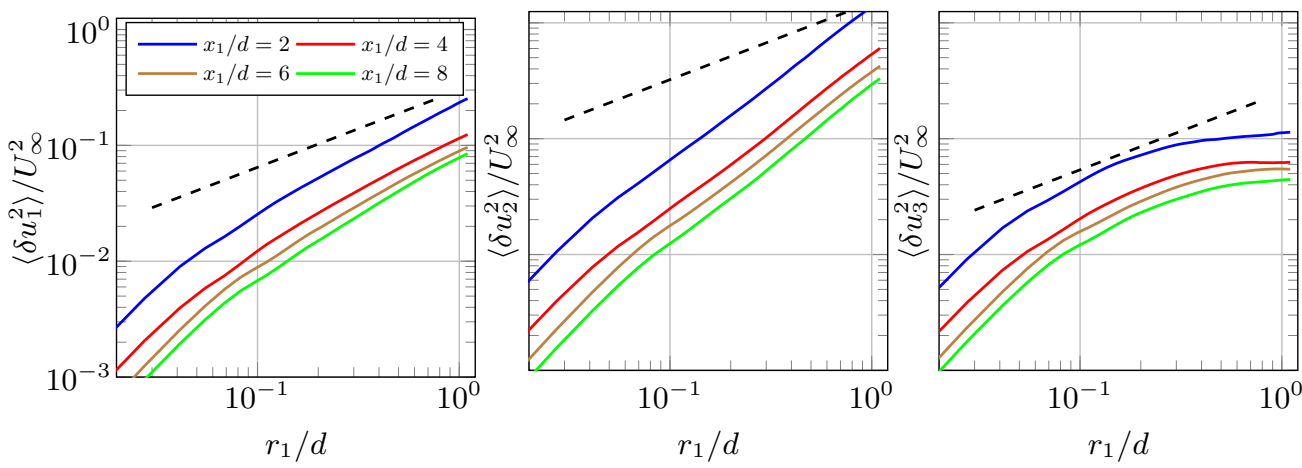

Figure 12: Second order structure functions of the three different velocity components along $r_{1}$ at three different locations on the centreline. The dashed line indicates a slope of $2 / 3$.

\subsection{Second Order Structure Functions and corresponding spectra}

Our supporting evidence for the Taylor hypothesis is limited to spatial separations $r_{1}$ in the streamwise direction and cannot be expected to be valid in other directions. We therefore extract from our DNS data second order structure functions $\left\langle\delta u_{i}^{2}\right\rangle$ for $i=1,2,3$ as functions of $\left(r_{1}, 0,0\right)$ at various locations $x_{1}$ on the centreline. Given the near $-5 / 3$ power law scalings observed in the frequency spectra, one might expect these second order structure functions to have a near $2 / 3$ power law dependence on $r_{1}$ in an intermediate range of scales. The power law range in the frequency spectra is, at most, about $0.5<f d / U_{\infty}<3$. Assuming that we can apply Taylor's hypothesis and therefore $r_{1} f=U_{c}$, this power law range of frequencies translates to $\frac{1}{3} U_{c} / U_{\infty}<r_{1} / d<2 U_{c} / U_{\infty}$, i.e. typically around $0.25<r_{1} / d<1.5$. However, as seen in fig. 12 , the structure functions $\left\langle\delta u_{i}^{2}\right\rangle$ (for $i=1,2,3$ ) do not have a near $2 / 3$ power law in this range. In fact, the approximate slopes of $\log \left(\left\langle\delta u_{1}^{2}\right\rangle\right)$ and $\log \left(\left\langle\delta u_{2}^{2}\right\rangle\right)$ vastly exceed $2 / 3$, while the $\log \left(\left\langle\delta u_{3}^{2}\right\rangle\right)$ curve reaches it only tangentially in this range.

The striking difference between the planar structure functions $\left\langle\delta u_{1}^{2}\right\rangle$ and $\left\langle\delta u_{2}^{2}\right\rangle$ and the spanwise structure function $\left\langle\delta u_{3}^{2}\right\rangle$ as well as the very steep growth of the planar structure functions compared to $r_{1}^{2 / 3}$ can be explained in terms of the vortex shedding which is clearly visible in figs. 9a and 10a but absent in fig. 10b. The lack of a clear 2/3 power law in the $r_{1}$ dependence of $\left\langle\delta u_{3}^{2}\right\rangle$ is consistent with the observation sometimes made (Frisch 1995; Lindborg 1999) that the range of wavenumbers (or frequencies) over which a well-defined $-5 / 3$ power law is observed is in general larger than the range of scales over which a $2 / 3$ power law for the second order structure is clearly present (see also Antonia et al. 2003). We now illustrate these two points in terms of a simple example.

We consider a model spectrum (see Pope 2000) given by

$$
E_{u}(k)=E_{0}(k) k^{-\frac{5}{3}} E_{\eta}(k)
$$

where $E_{0}(k)$ and $E_{\eta}(k)$ characterise, respectively, the low and high wavenumber behaviour of the spectrum and are constant in the intermediate range of wavenumbers $k$ where $E_{u}(k) \propto k^{-\frac{5}{3}}$. We take functions $E_{0}(k)$ and $E_{\eta}(k)$

$$
E_{0}(k)=\left(\frac{k / k_{0}}{\left[\left(k / k_{0}\right)^{2}+c_{0}\right]^{\frac{1}{2}}}\right)^{5 / 3}
$$



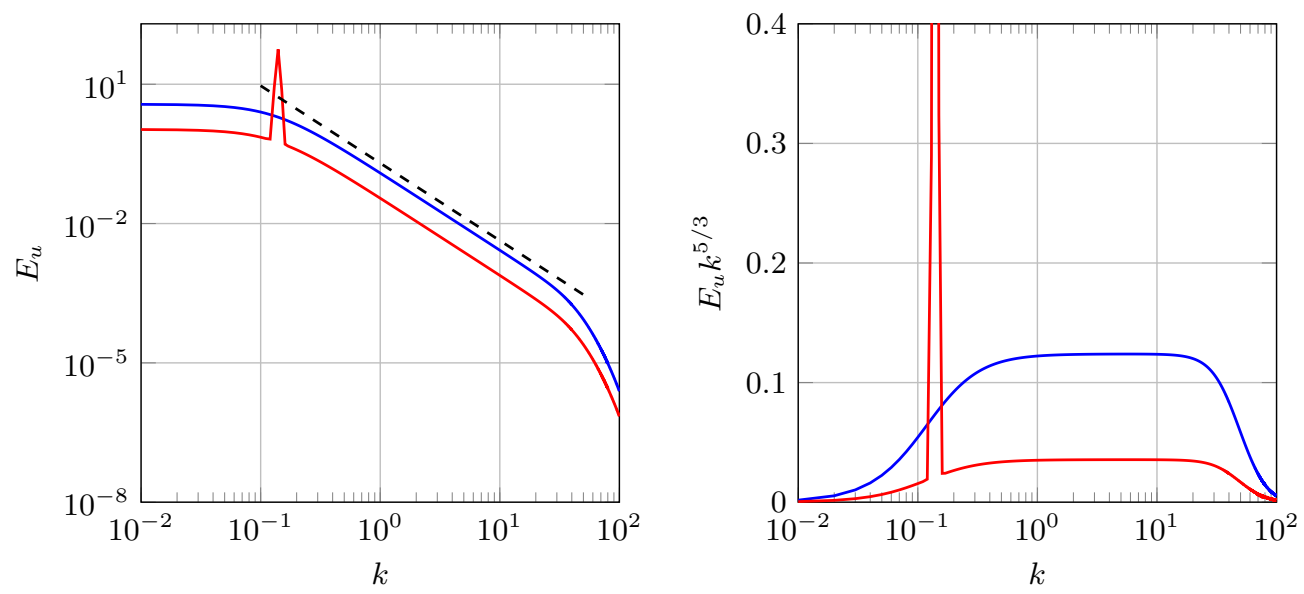

Figure 13: Models of a spectrum with one decade inertial range. One of the spectra (in red) is amplified at a given wavenumber. On the right the spectra are compensated by $k^{5 / 3}$.

$$
E_{\eta}(k)=\exp \left(-c_{\eta} k / k_{\eta}\right)
$$

so that $E_{u}(k) \propto k^{-\frac{5}{3}}$ in the range $k_{0} \ll k \ll k_{\eta}$. We chose the constants $c_{0}$ and $c_{\eta}$ and the inner and outer wavenumbers $k_{0}$ and $k_{\eta}$ such that the spectrum takes the form shown infig. 13 In the same figure we also plot another model spectrum obtained in the exact same way but with highly amplified energy around a wavenumber $k_{s}$ (both spectra integrate to the same total kinetic energy). We do so via

$$
E_{s}(k)=E(k)\left\{1+A \exp \left[\frac{-\left(k-k_{s}\right)^{2}}{2 \Delta k_{s}^{2}}\right]\right\}
$$

where $A$ is an amplification factor, $k_{s}$ is the wavenumber associated with the shedding and $\Delta k_{s}$ represents the spread of the spectral peak. Equation (3.4) attempts to mimic the model introduced in Thiesset et al. (2014) in scale space which is effectively associated with a peak in spectral space. The $-5 / 3$ power law is well defined for over one decade of wavenumbers in both model spectra as made clear in the second plot of fig. 13 where the spectra have been compensated by $k^{5 / 3}$.

We now calculate the second order structure functions corresponding to the two model spectra of fig. 13 from

$$
\left\langle\delta u^{2}\right\rangle=2 u^{\prime 2}\left[1-\mathcal{F}^{-1}\left(E_{u}\right)\right]
$$

where $\mathcal{F}^{-1}$ denotes an inverse Fourier transform. These structure functions are plotted in fig. 14 and one can make two clear observations. Firstly, there is no $2 / 3$ power law even though there is a well-defined $-5 / 3$ power law in the spectrum from which these structure functions are derived. At best, $\left\langle\delta u^{2}\right\rangle$ reaches $r^{2 / 3}$ only tangentially in the case of the model spectrum without the shedding peak. This is similar to our result plotted in fig. 12 for $\left\langle\delta u_{3}^{2}\right\rangle$ which concerns spanwise fluctuating velocities unaffected by the planar vortex shedding. Secondly, the vortex shedding peak of one of the two model spectra has introduced a growth much steeper than a $2 / 3$ power law in the corresponding structure function. As noted by Thiesset et al. (2014), one should indeed expect the effect of the shedding on the second order structure function to be reflected by a sinusoid-like function; fig. 14 demonstrates that this sinusoid can, in fact, very significantly contaminate the power law range of the structure function even when the corresponding energy spectrum 

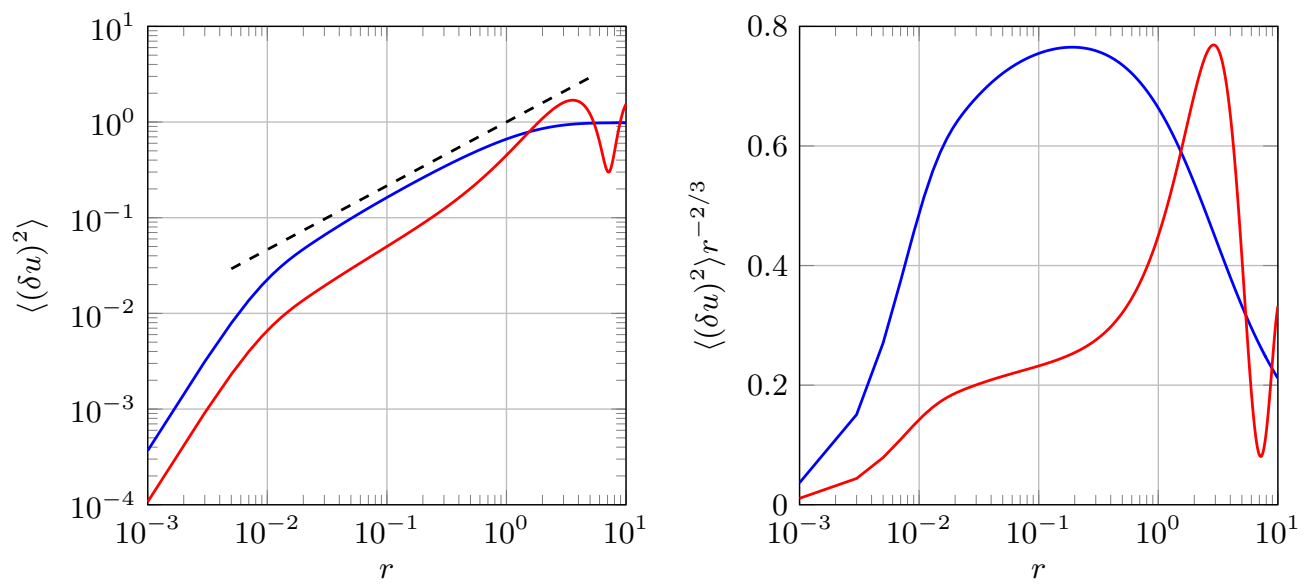

Figure 14: Second order structure functions obtained by the inverse Fourier transform of the spectra plotted in fig. 13 . On the right the structure functions are compensated by $r^{-2 / 3}$.

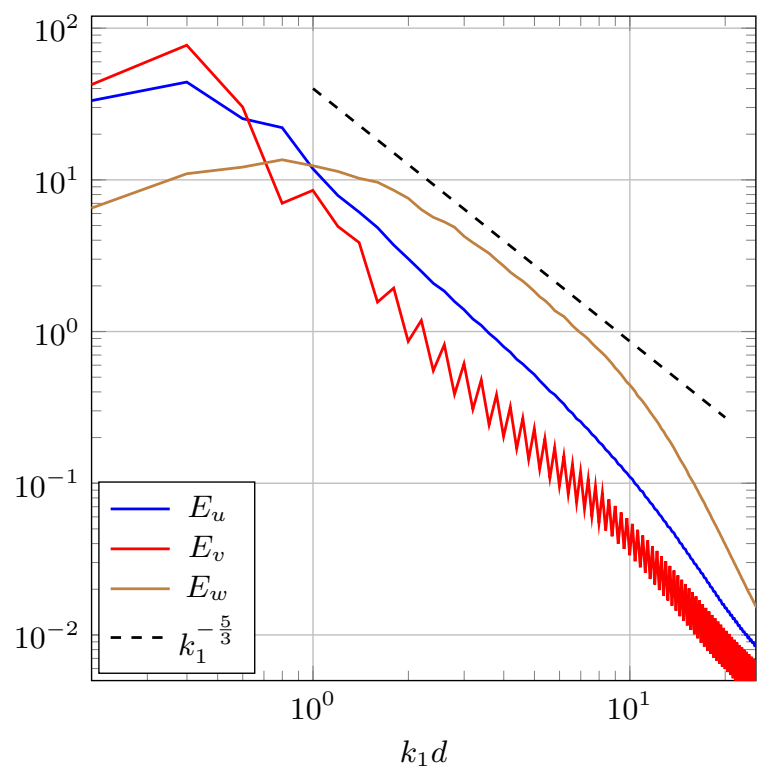

Figure 15: Energy spectra in wavenumber obtained by Fourier transform of the correlation functions of each velocity component at $x_{1} / d=4$.

has a well-defined $k^{-5 / 3}$ range over more than a decade. The resulting structure function is much steeper than a $2 / 3$ power law in the range of scales which corresponds to the $-5 / 3$ part of the spectrum. This is similar to our results plotted in fig. 12 for $\left\langle\delta u_{1}^{2}\right\rangle$ and $\left\langle\delta u_{2}^{2}\right\rangle$ which concerns planar fluctuating velocities which are affected by vortex shedding.

Inverting (3.5) and using it to obtain the spectra which correspond to the structure functions in fig. 12 does return $-5 / 3$ power law spectra over a range of wavenumbers comparable to the range of frequencies where figs. 9a, 10a and $10 \mathrm{~b}$ exhibit $-5 / 3$ power laws (see figure fig. 15).

In conclusion, the evidence presented in this and the previous subsections supports 
(a)
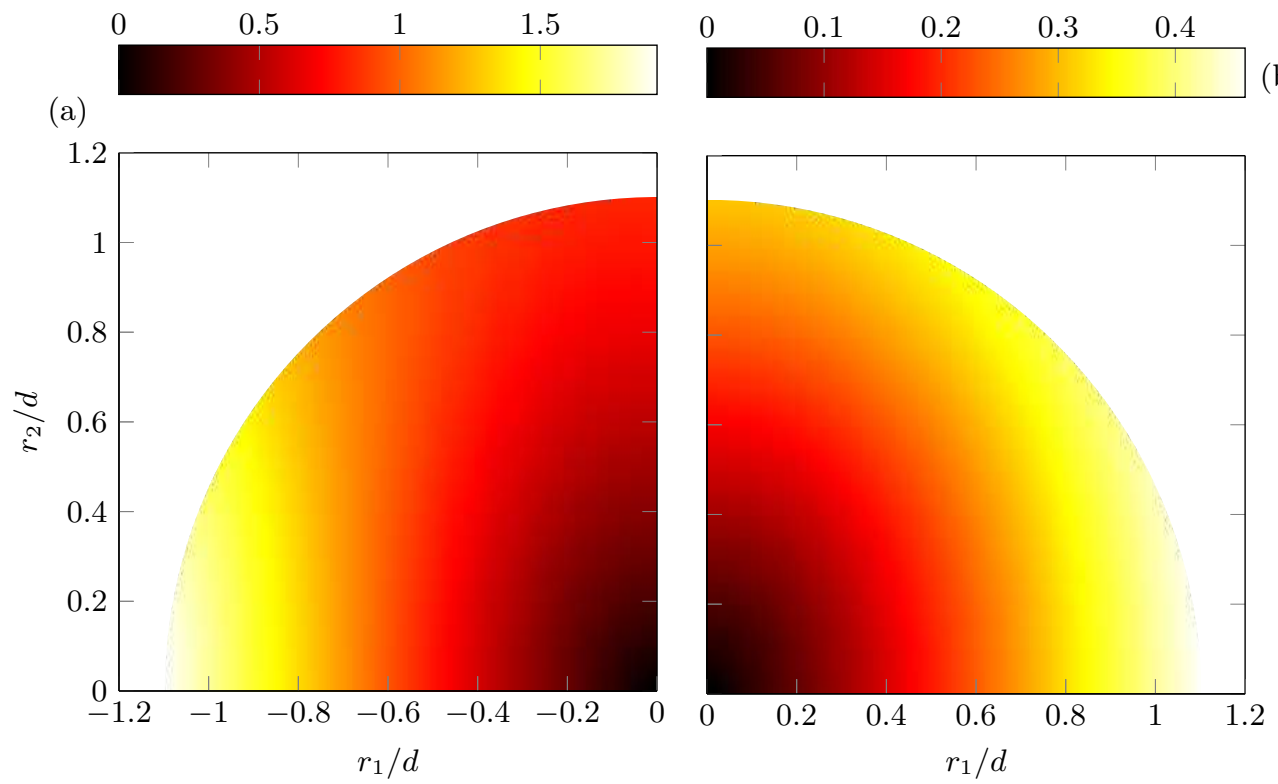

Figure 16: Distribution of $\left\langle\delta q^{2}\right\rangle$ normalised by $U_{\infty}^{2}$ in scale space on the geometrical centreline at $x_{1} / d=2$ and $x_{1} / d=8$ in (a) and (b), respectively.

the view that there is an underlying approximate $r_{1}^{2 / 3}$ dependence in all three second order structure functions on the centreline of our DNS but over a very short $r_{1}$-range. In the case of the streamwise and cross-stream structure functions, this range is totally overshadowed by the vortex shedding signature. The energy spectrum amplifies the power law region and makes it appear as a clear near $-5 / 3$ power law over a wide range of frequencies/wavenumbers while at the same time disentangling it from the vortex shedding signature. However these near $-5 / 3$ power law spectra cannot be obviously explained by the Kolmogorov theory: their range appears to reduce rather than increase with Reynolds number and, as we show in the following subsection, there is no isotropy in that range.

\subsection{Second order structure functions and isotropy}

In fig. $16\left\langle\delta q^{2}\right\rangle$ is plotted for $r_{3}=0$ at two different locations in space. The distribution of $\left\langle\delta q^{2}\right\rangle$ in scale-space shows that the energy is distributed anisotropically in the range $0.2 \leqslant r / d$ which includes most of the range $0.15<r_{1} / d<1.5$ where the $-5 / 3$ power laws are observed. Comparing fig. 16a with fig. 16b shows that there is an overall decrease of the energy and suggests some tendency towards isotropy with increasing downstream position.

The structure functions associated with each velocity component at $x_{1} / d=2$ are shown in fig. 17. It is clear that the large values of $\left\langle\delta q^{2}\right\rangle$ observed in fig. 16 are associated with the large contribution of $\left\langle\delta u_{2}^{2}\right\rangle$ compared to the other two components. In fact, one could have anticipated that, at least for orientations aligned with the mean flow direction, the structure function associated with the vertical fluctuations would have a significant contribution to the total energy, as comparing fig. 6 with figs. 5 and 7 shows that it is that component which contributes the most to the turbulent kinetic energy.

The plots in fig. 17 show very distinct anisotropy except for $\left\langle\delta u_{3}^{2}\right\rangle$ which is unaffected by the vortex shedding. As seen at the start of this section, the frequency spectra of 

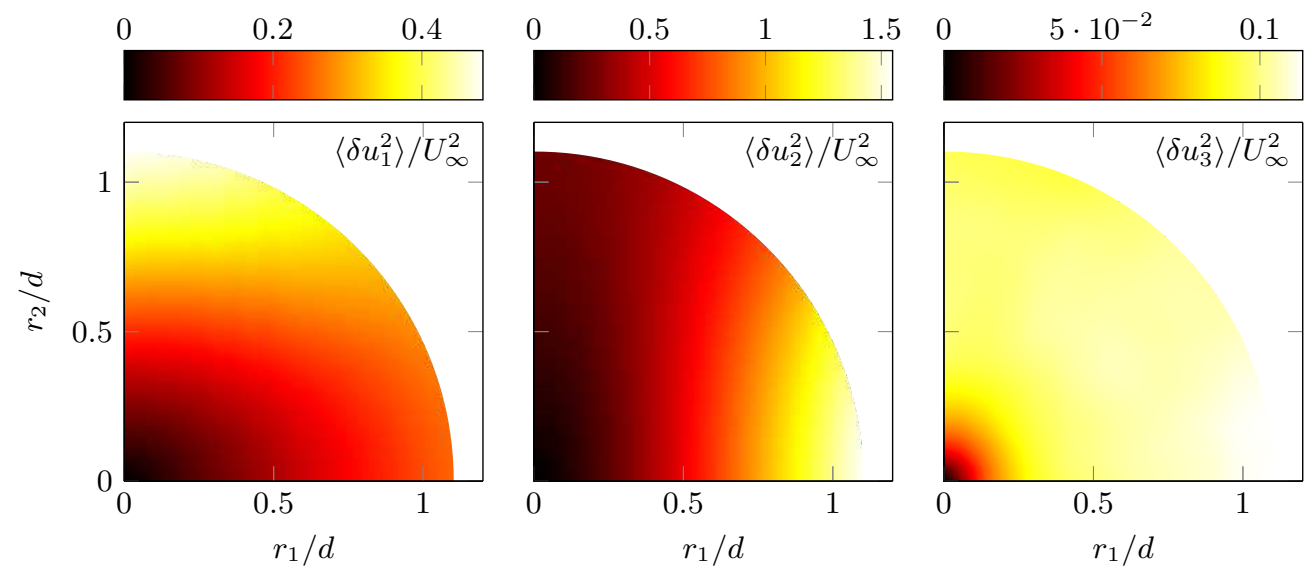

Figure 17: $i$-th component of the second order structure function $\left\langle\delta u_{i}^{2}\right\rangle$ normalised by $U_{\infty}^{2}$ on the centreline at $x_{1} / d=2$ on the centreline

$u_{1}$ and $u_{2}$ show a persistent peak at the frequency associated with the vortex shedding, indicating that the anisotropy observed in fig. 16 and fig. 17 is most likely due to the coherent motion as also suggested by Thiesset et al. $(2013 \mathrm{~b})$. However it is not easy with the tools used in this paper to disentangle the shedding-induced anisotropy from an underlying potentially isotropic small-scale turbulence which may or may not be amenable to the Kolmogorov framework. We leave this issue for a future study based on a triple decomposition of the velocity field which distinguishes between coherent and incoherent fluctuations. In the following section we proceed with the study of the various terms in the KHMH equation which in fact brings further, and in fact more substantial, evidence of anisotropy.

\section{Scale-by-scale Budget of $\left\langle\delta q^{2}\right\rangle$}

\subsection{Forward and Inverse Cascades by Non-linear Interactions}

The non-linear inter-scale transfer rate $\Pi$ is the divergence in scale space of the nonlinear inter-scale flux vector $\left\langle\delta \mathbf{u} \delta q^{2}\right\rangle$. The orientation of this flux is directly linked to the concept of energy cascade as it can characterise the direction where energy flows in scale space. In the context of the Richardson-Kolmogorov equilibrium cascade and the assumption of local isotropy, this flux has a radial component which points from large to small separations for any orientation.

From now on we concentrate attention on four centreline locations, namely $x_{1} / d=$ $2,4,6,8$, all far enough from the recirculation region and from the downstream end of our DNS domain, see figs. 2 and 4 . In fig. 18 we plot the non-linear inter-scale flux in scale space at two centreline locations. The figures show the direction of the vector $\left\langle\delta \mathbf{u} \delta q^{2}\right\rangle$ with its magnitude in the background. While the magnitude of $\left\langle\delta \mathbf{u} \delta q^{2}\right\rangle$ is, in general, larger at large separations, it's distribution in scale space is far from isotropic. Moreover, the orientation of $\left\langle\delta \mathbf{u} \delta q^{2}\right\rangle$ varies drastically with the orientation of the scale vector $\mathbf{r}$, suggesting that $\left\langle\delta q^{2}\right\rangle$ is, on average, redistributed in scale space, rather than solely transferred from large to small $r$.

Notice how, at $x_{1}=2$ the flux is directed outwards (from small to large $r / d$ ) at separations close to the $r_{2}=0$ axis. At a location further downstream $\left(x_{1} / d=8\right)$ the fluxes have rotated by $90 \mathrm{deg}$ and the flux is now directed inwards (from large to small 

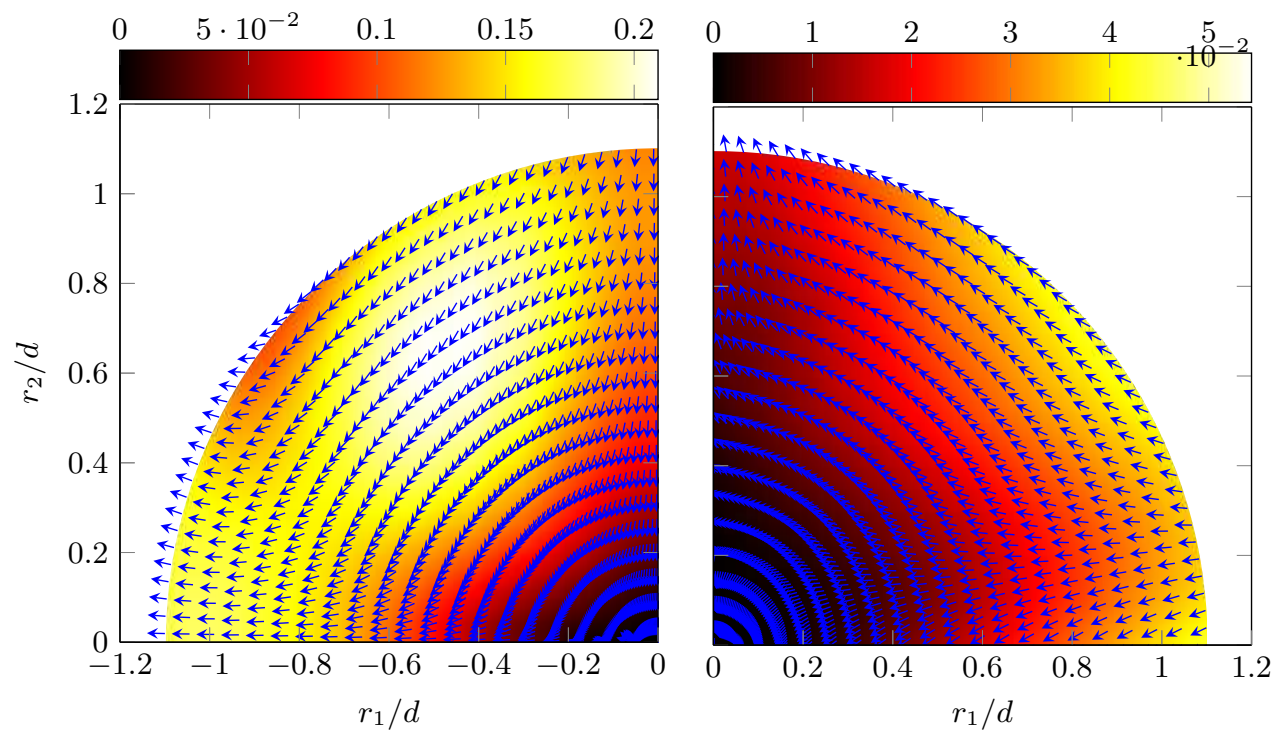

Figure 18: Inter-scale flux of energy due to non-linear interactions $\left\langle\delta \mathbf{u} \delta q^{2}\right\rangle$ at $x_{1} / d=2$ (left) and $x_{1} / d=8$ (right) for $r_{3}=0$. The arrows indicate the orientation of the vector $\left\langle\delta \mathbf{u} \delta q^{2}\right\rangle$ and the contour indicates the magnitude normalised by $U_{\infty}^{3}$.

$r / d$ ) at separations close to the $r_{2}=0$ axis (in agreement with the measurements of Thiesset et al. (2014)) but outwards at separations close to the $r_{1}=0$ axis.

A necessary condition for a forward or inverse cascade is that the radial component

$$
\left\langle\delta u_{r} \delta q^{2}\right\rangle=\left\langle\delta u_{i} \delta q^{2}\right\rangle r_{i} / r
$$

of the flux $\left\langle\delta \mathbf{u} \delta q^{2}\right\rangle$ is positive or negative.

While the sign of $\left\langle\delta u_{r} \delta q^{2}\right\rangle$ indicates if the flux is from large to small (negative) or small to large (positive) scales, it is not enough to characterise the energy cascade as being direct or inverse. For the energy to be truly cascading from large to small scales it is necessary that negative values of $\left\langle\delta u_{r} \delta q^{2}\right\rangle$ be associated with negative values of the radial component of the non-linear inter-scale transfer rate $\Pi$ written in cylindrical coordinates (with the cylindrical axis in the spanwise direction) i.e.

$$
\Pi_{r}=\frac{1}{4} \frac{\partial}{\partial r}\left\langle\delta u_{r} \delta q^{2}\right\rangle+\frac{1}{4 r}\left\langle\delta u_{r} \delta q^{2}\right\rangle .
$$

Conversely, for the energy to be cascading from small to large scales positive values of $\left\langle\delta u_{r} \delta q^{2}\right\rangle$ must be associated with positive values of $\Pi_{r}$. In other words, the radial flux $\left\langle\delta u_{r} \delta q^{2}\right\rangle$ is only responsible for an accumulation (depletion) of energy at a given separation vector $\mathbf{r}$ if the gradient given by eq. (4.2) is positive (negative) at that separation vector. Figures 19 and 20 map eqs. (4.1) and (4.2) in scale space at the same locations as fig. 18 .

It is now clear that figs. 19 and 20] suggest a co-existence of forward and inverse nonlinear cascades at both centreline locations $x_{1} / d=2$ and $x_{1} / d=8$. In agreement with fig. 18, the orientations where these two simultaneous cascades operate differ at these two locations. The cascade appears inverse in the streamwise and forward in the cross stream direction at $x_{1} / d=2$ but forward in the streamwise and inverse in the cross stream direction at $x_{1} / d=8$. It is important to note that these local, in scale space, inverse cascade behaviours coexist with energy spectra characterised by near $-5 / 3$ power laws, in 

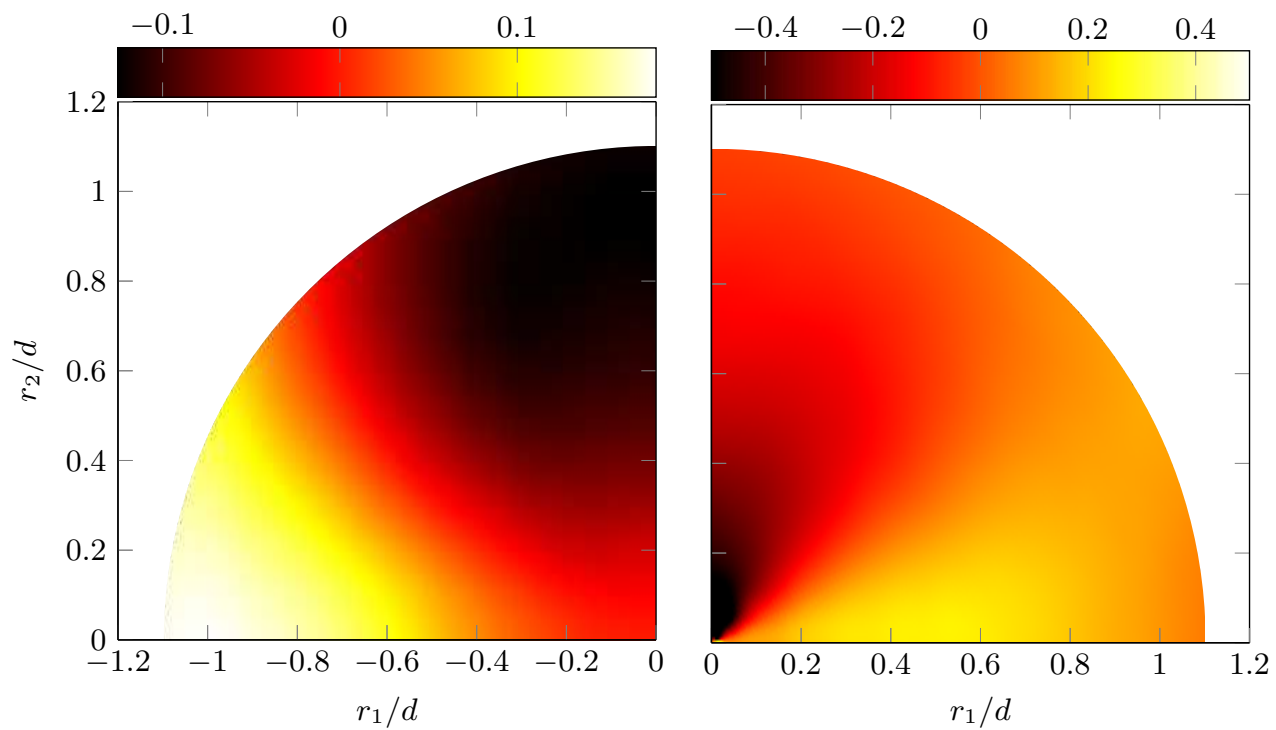

Figure 19: Radial contributions to the non-linear interscale flux (left) and transfer (right) of $\left\langle\delta q^{2}\right\rangle$ at $x_{1} / d=2$, eqs. (4.1) and (4.2) respectively.
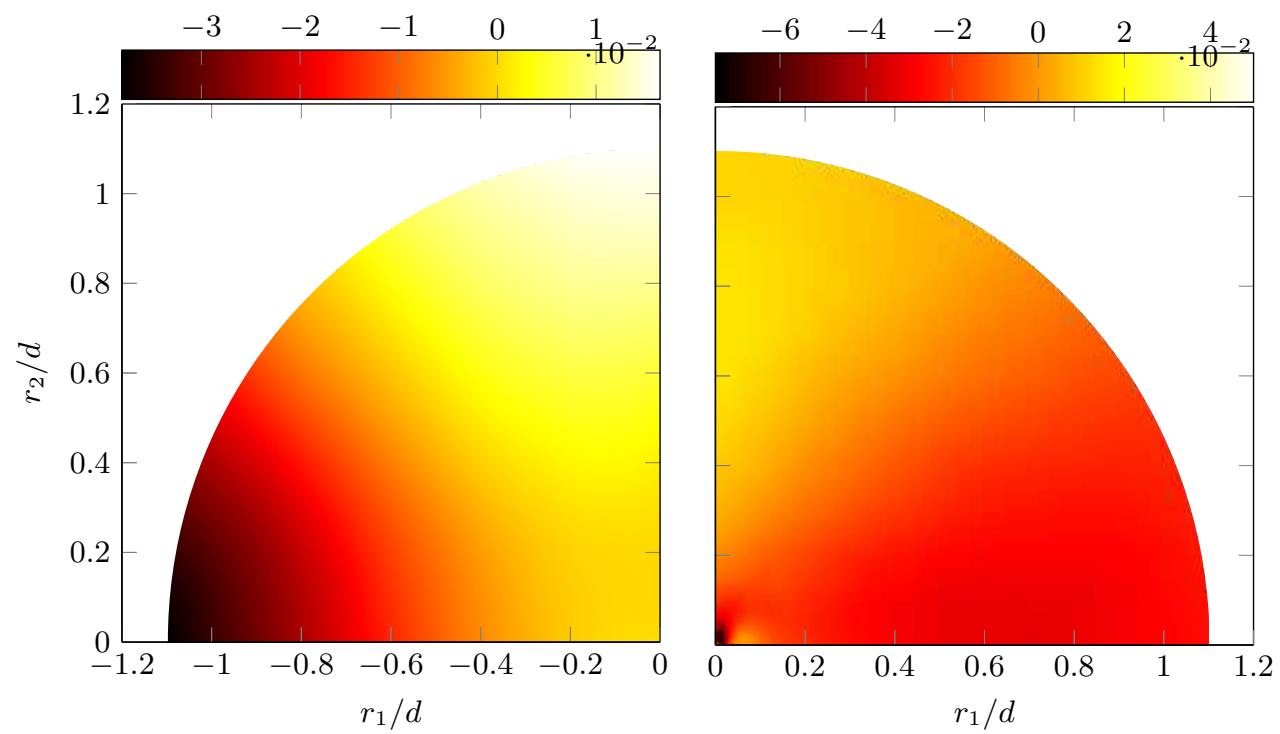

Figure 20: Radial contributions to the non-linear interscale flux (left) and transfer (right) of $\left\langle\delta q^{2}\right\rangle$ at $x_{1} / d=8$, eqs. (4.1) and (4.2) respectively.

particular at $x_{1} / d=2$ where these power laws are closest to $-5 / 3$ and the inverse cascade behaviour is in the streamwise direction where the spectra are effectively evaluated. Such coexistence as the one at $x_{1} / d=2$ was already observed in Gomes-Fernandes et al. (2015) in the very near field of a different turbulent flow, while the experiments of Thiesset et al. (2014) did not allow capturing these phenomena because their measurements where carried out further downstream and because their measurement apparatus was limited to $r_{2}=0$.

Given the presence of simultaneous forward and inverse cascade behaviours along 


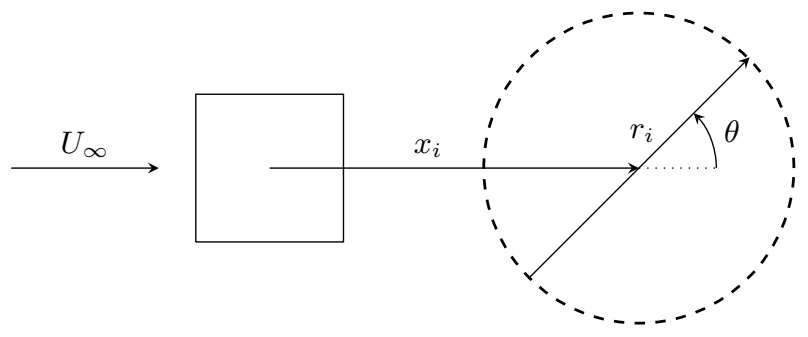

Figure 21: Illustration of the $r_{3}=0$ plane for $x_{i}$ aligned with the centreline. The angle $\theta$ indicates the angle over which the orientations of $r_{i}$ are averaged.

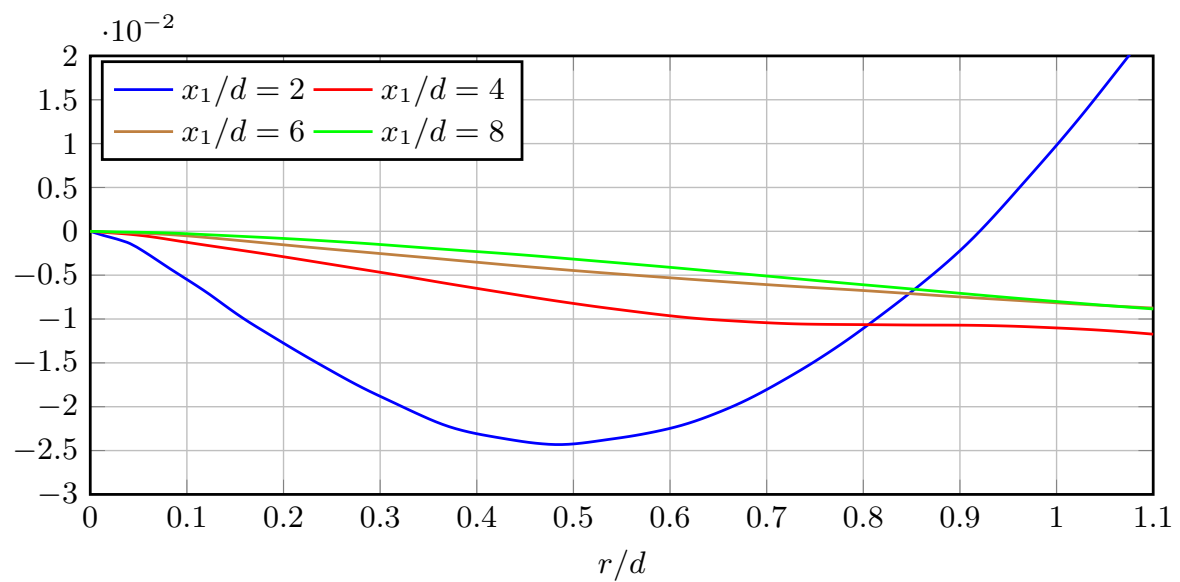

Figure 22: Orientation averaged radial component of the non-linear inter-scale flux $\left\langle\delta u_{r} \delta q^{2}\right\rangle^{a}$ at four different locations along the centreline.

different scale space orientations, it is important to average over these orientation angles (as illustrated in fig. 21) and work out if the non-linear cascade is, on average, forward or inverse. The orientation averaged radial component of the non-linear inter-scale flux, i.e. $\left\langle\delta u_{r} \delta q^{2}\right\rangle^{a}=\frac{1}{2 \pi} \int_{0}^{2 \pi} d \theta\left\langle\delta u_{r} \delta q^{2}\right\rangle$ (where $\theta$ is the scale space orientation angle, i.e. $r_{1} / r=\cos \theta$, seefig. 21), is a function of $r$ and is plotted in fig. 22 for centreline locations $x_{1} / d=2,4,6,8$. Apart from the location closest to the the cylinder $\left(x_{1} / d=2\right)\left\langle\delta u_{r} \delta q^{2}\right\rangle^{a}$ remains negative over all scales investigated and even at $x_{1} / d=2$ it remains negative over a wide range of scales, $r / d<0.86$. This means that the non-linear cascade is forward on average on the centreline near field $2 \leqslant x_{1} / d \leqslant 8$ of a square prism's turbulent wake, and results from combined forward and inverse cascades in different directions as exemplified by figs. 19 and 20.

We distinguish between "forward/inverse cascades" and "forward/inverse cascade behaviours" because the possibility remains that turbulent flow inhomogeneities, in particular at the larger scales but a priori even potentially at some smaller scales, may have some contribution to the behaviour of the interscale flux vector $<\delta \mathbf{u} \delta q^{2}>$. Equally, inhomogeneity may be a priori and at least partly responsible for the predominantly negative values of $\left\langle\delta u_{r} \delta q^{2}\right\rangle^{a}$ indicating a forward cascade on average. Untangling the inhomogeneity from the actual cascade contributions is a delicate and important question which will need to be carefully addressed in future works. 

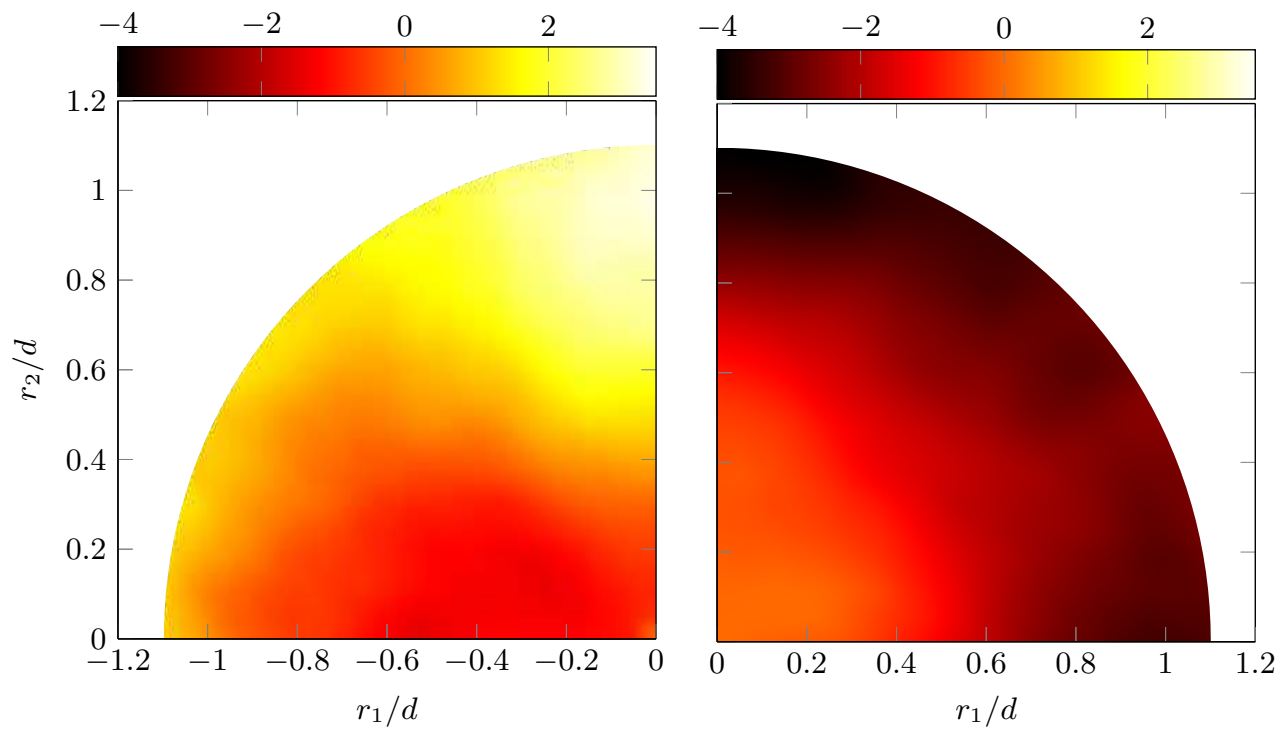

Figure 23: Non-linear interscale transfer $\Pi$ (left) and pressure term $\mathcal{T}_{p}$ (right) normalised by $\varepsilon_{r}$ at $x_{1} / d=2$ on the centreline.

\subsection{Contributions of the Different Terms in the KHMH Equation}

As the KHMH equation clearly illustrates, the non-linear cascade does not happen in isolation unless the length-scales considered are so small that direct viscous diffusion effects matter and/or the turbulence is sufficiently inhomogeneous for at least one of the following, production $\mathcal{P}$, linear inter-scale transfer rate $\Pi_{U}$, spatial transport $\mathcal{T}_{u}$ and pressure term $\mathcal{T}_{p}$, to be significant. The type of inhomogeneity which causes the advection term $\mathcal{A}$ to be non-negligible when averaging is over time and $\mathcal{A}_{t}=0$ by construction, is a reflection of the unsteady nature of the small-scale turbulence in the frame following a mean flow. In this subsection we examine all these additional processes in order to establish the energy exchange context in which the previous section's non-linear cascade occurs.

We first confirmed that the viscous terms $\mathcal{D}_{x}$ and $\mathcal{D}_{r}$ are indeed negligible in (2.1), except at very small scales (comparable to or smaller than the local Taylor microscale which is typically around $d / 10$ at the centreline positions investigated) (see Appendix B in Valente \& Vassilicos 2015). We therefore now deal with $\Pi$ and $\mathcal{T}_{p} ; \mathcal{A}$ and $\mathcal{T}_{u}$; and $\mathcal{P}$ and $\Pi_{U}$.

In figs. 23 and 24 both $\Pi$ and $\mathcal{T}_{p}$ are plotted in scale space at two different locations on the centreline $\left(x_{1} / d=2\right.$ and $x_{1} / d=8$, respectively). In section $2.1 \mathcal{T}_{p}$ is written as $-\frac{1}{2} \frac{\partial\left\langle\delta u_{i} \delta p\right\rangle}{\partial x_{i}}$; however, another possible formulation is $-\frac{1}{4} \frac{\partial\left\langle\delta u_{i}\left(p^{+}+p^{-}\right)\right\rangle}{\partial r_{i}}$ which resembles the expression for $\Pi$ with $-\left(p^{+}+p^{-}\right)$taking the place of $\delta q^{2}$. At both locations $\left(x_{1} / d=2\right.$ and $\left.x_{1} / d=8\right), \Pi$ acts simultaneously as a source $(\Pi<0)$ and a sink term $(\Pi>0)$ in eq. (2.1). However, $\mathcal{T}_{p}$ is initially (at $\left.x_{1} / d=2\right)$ a sink term $\left(\mathcal{T}_{p}<0\right)$ at all orientations and amplitudes of $\mathbf{r}$ while further downstream (at $x_{1} / d=8$ ), just like $\Pi$, it acts as both a source $\left(\mathcal{T}_{p}>0\right)$ and a $\operatorname{sink}$ term $\left(\mathcal{T}_{p}<0\right)$ in eq. $(2.1)$

It is important to distinguish $\Pi$ from $\Pi_{r}$ (see eq. (4.2) shown in figs. 19 and 20 . In section 4.1, $\Pi_{r}$ is used to describe how $\delta q^{2}$ is exchanged from large to small scales (and vice versa). However, the term which actually appears in the budget of $\left\langle\delta q^{2}\right\rangle$ is $\Pi$, the divergence in scale space of the non-linear interscale flux $\left\langle\delta \mathbf{u} \delta q^{2}\right\rangle$. Figures 23 and 24 

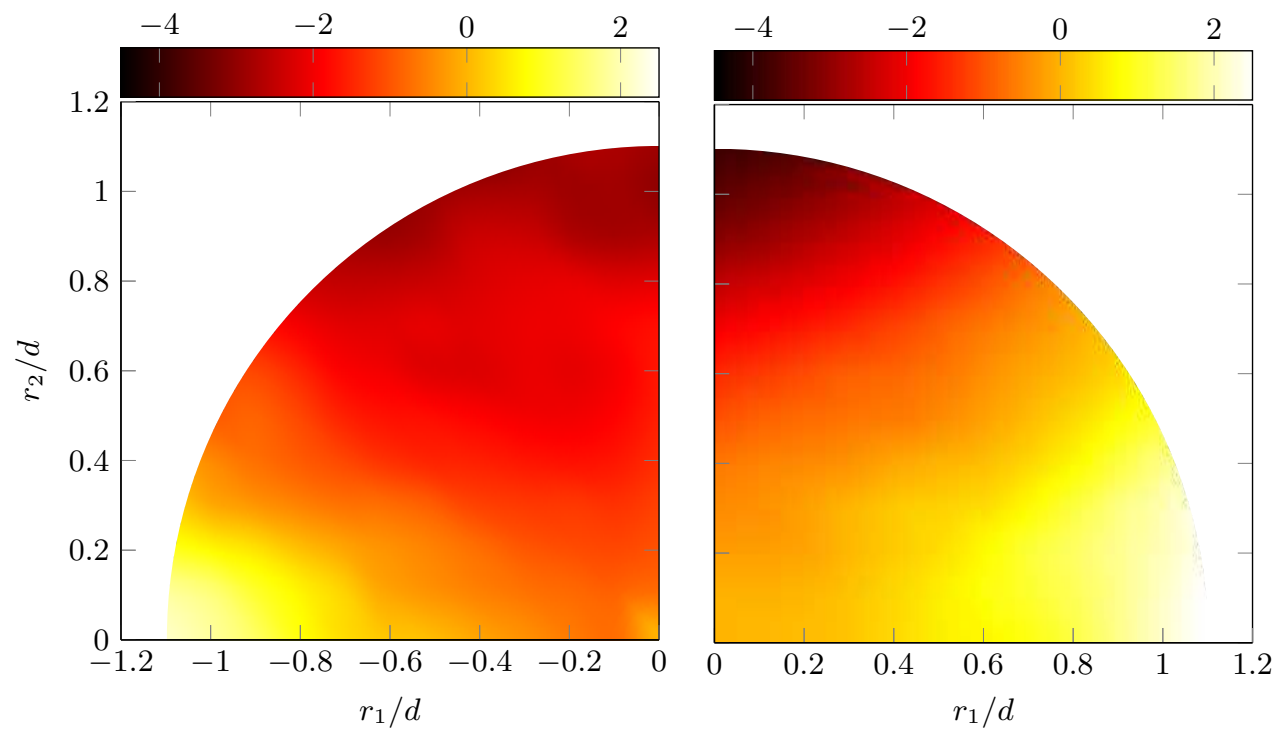

Figure 24: Non-linear interscale transfer $\Pi$ (left) and pressure term $\mathcal{T}_{p}$ (right) normalised by $\varepsilon_{r}$ at $x_{1} / d=8$ on the centreline.

reveal that the non-linear interactions can act as a source of $\left\langle\delta q^{2}\right\rangle$ independently from the orientation of $\left\langle\delta u_{i} \delta q^{2}\right\rangle$, i.e., it is not necessary to have a forward cascade $\left(\left\langle\delta u_{r} \delta q^{2}\right\rangle<0\right)$ to observe $\Pi<0$.

What is also, perhaps, surprising from figs. 23 and 24 is how $\mathcal{T}_{p}$ looses its somewhat isotropic distribution as $x_{1}$ increases. Moreover, it would appear from fig. 24 that there is some correlation at $x_{1} / d=8$ between the distribution of $\mathcal{T}_{p}$ and $\Pi$ in scale space, in agreement with recent results by Yasuda \& Vassilicos (2017) for periodic turbulence. The fact that $\mathcal{T}_{p}$ remains a significant term in the KHMH equation may not be fully unexpected, from the measurements of Gomes-Fernandes et al. (2015) for example, but it does not seem to have been previously reported. One may anticipate that $\mathcal{T}_{p}$ reflects the presence of the coherent vortices associated with the shedding which introduce strong pressure gradients in the flow.

Two other terms which also appear to correlate as downstream location increases are the terms responsible for transporting $\delta q^{2}$ in physical space: $\mathcal{A}$ and $\mathcal{T}_{u}$. In figs. 25 and 26 these two terms are shown side by side for $x_{1} / d=2$ and $x_{1} / d=8$ respectively. At all separation vectors $\mathbf{r}$ considered one can observe that $\mathcal{A}<0$. This indicates that, regardless of the orientation and amplitude of $\mathbf{r},\left\langle\delta q^{2}\right\rangle$ decays in the direction of the mean flow. $\mathcal{T}_{u}$ also appears to be predominantly negative, especially at the location closest to the prism.

At $x_{1} / d=2 \mathcal{A}$ appears to be roughly independent of the orientation of $r_{i}$ suggesting that initially the decay of $\left\langle\delta q^{2}\right\rangle$ depends only on $r=\left|r_{i}\right|$. However, as $x_{1}$ increases and the production term weakens, $\mathcal{A}$ appears to tend to map in scale space in a way roughly similar to $\mathcal{T}_{u}$. This suggests the development with downstream distance of a sweeping mechanism (see Tsinober 2009) whereby small scale turbulent eddies are transported by the larger turbulent eddies so that a tendency develops for $\mathcal{A}$ and $\mathcal{T}_{u}$ to more or less balance.

The existence of a strong sweeping correlation between $\mathcal{A}$ and $\mathcal{T}_{u}$ is supported by the measurements of Gomes-Fernandes et al. (2015) which revealed an even stronger 
The Turbulence Cascade in the Near Wake
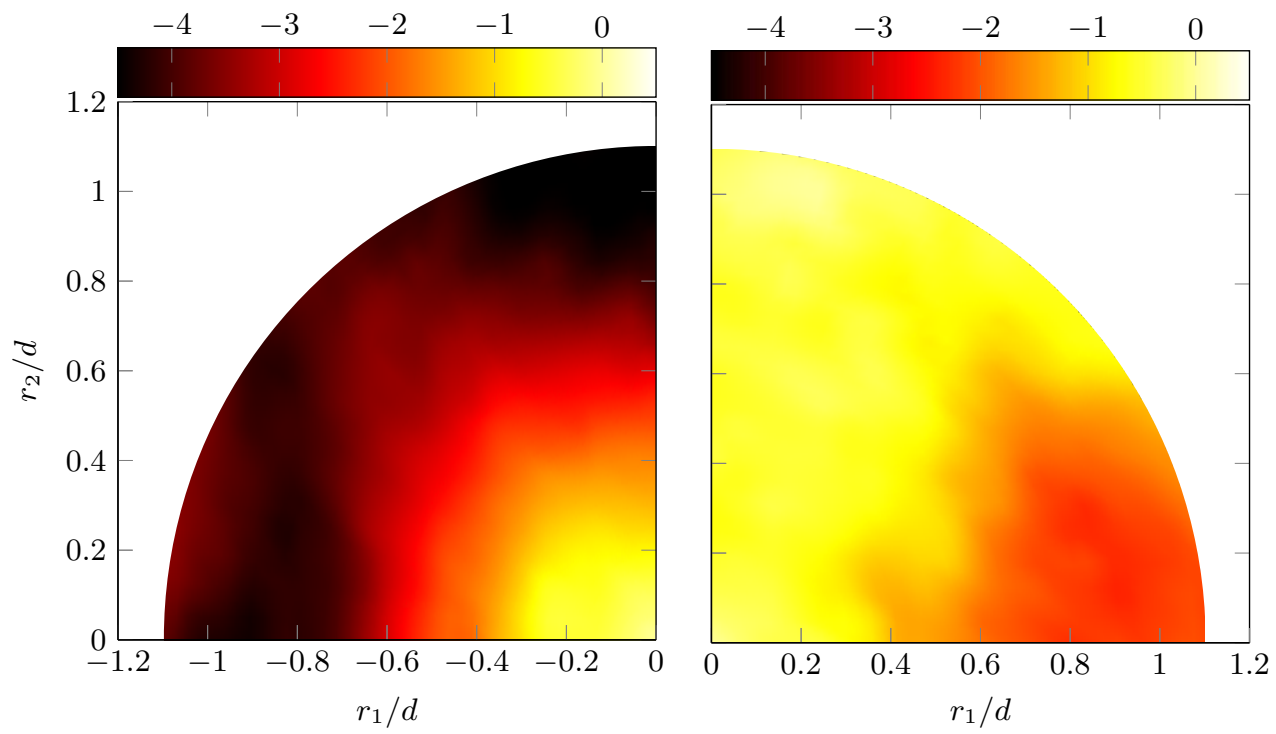

Figure 25: Advection $\mathcal{A}$ (left) and turbulent transport $\mathcal{T}_{u}$ (right) normalised by $\varepsilon_{r}$ at $x_{1} / d=2$ on the centreline.
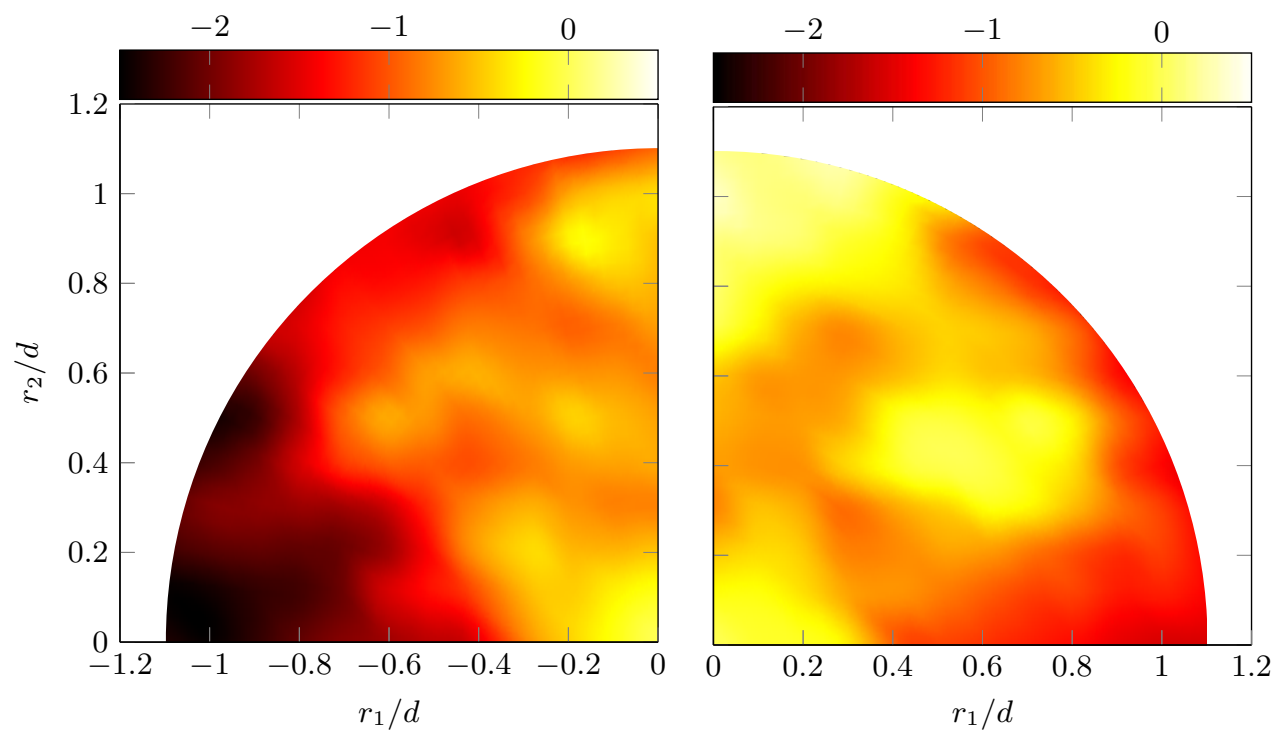

Figure 26: Advection $\mathcal{A}$ (left) and turbulent transport $\mathcal{T}_{u}$ (right) normalised by $\varepsilon_{r}$ at $x_{1} / d=8$ on the centreline.

correlation between $\mathcal{A}$ and $\mathcal{T}_{u}$ than evidenced here (see fig. 22 of that paper) albeit in a different turbulent flow configuration, and by the recent DNS of periodic turbulence by Yasuda \& Vassilicos (2017).

The two remaining terms to be considered in the KHMH equation are $\mathcal{P}$ and $\Pi_{U}$ which, unlike the previous terms which reflect inhomogeneity of the turbulence, are associated with inhomogeneity of the mean flow. One therefore expects their contribution in eq. (2.1) to decrease with increasing downstream location as is indeed observed by comparing fig. 27 and fig. 28 


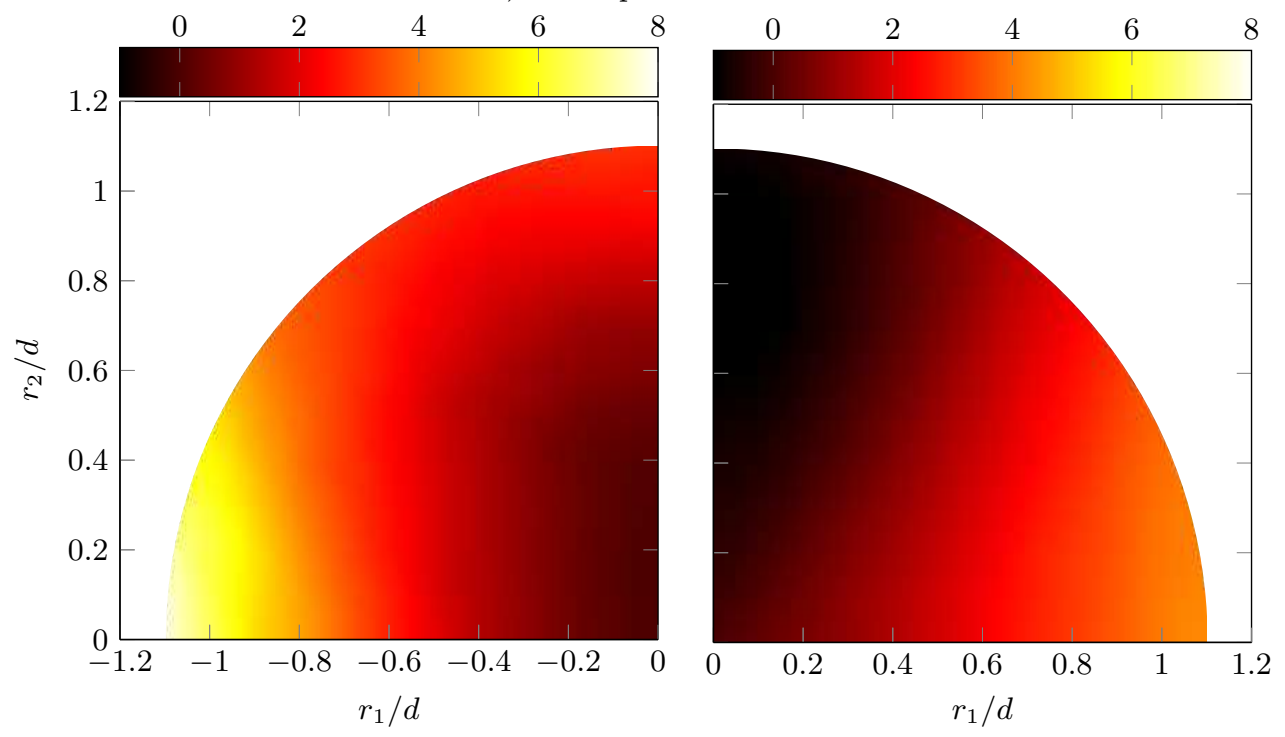

Figure 27: Production $\mathcal{P}$ (left) and linear interscale transfer $\Pi_{U}$ (right) normalised by $\varepsilon_{r}$ at $x_{1} / d=2$ on the centreline.
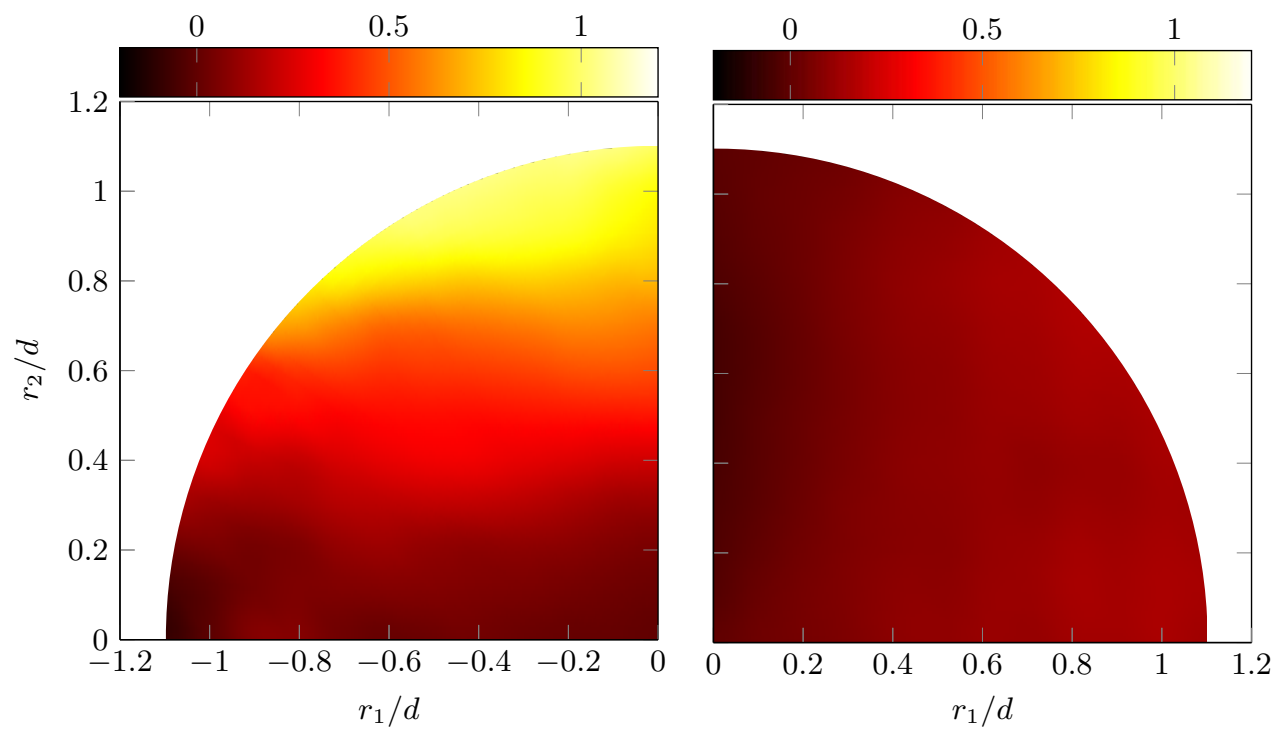

Figure 28: Production $\mathcal{P}$ (left) and linear interscale transfer $\Pi_{U}$ (right) normalised by $\varepsilon_{r}$ at $x_{1} / d=8$ on the centreline.

Apart from the clear decrease in magnitude, it is also clear that the distribution of $\mathcal{P}$ undergoes a reorientation in scale space, perhaps similar to the one reported in fig. 18 for the interscale flux. $\mathcal{P}$ appears stratified along the $r_{1}$ axis at $x_{1} / d=2$, presumably as a consequence of the strong mean flow gradients in the streamwise direction very close to the prism (see fig. 4). Further downstream, at $x_{1} / d=8, \mathcal{P}$ appears stratified along the $r_{2}$ axis. The largest variations of mean velocity occur in the cross-stream direction at that position.

It may be interesting to contrast the scale space maps of $\mathcal{P}$ in fig. 27 and fig. 28 and 
those of the the interscale flux in fig. 18. At both centreline locations, $x_{1} / d=2$ and $x_{1} / d=8$, the apparently inverse cascading interscale flux which points from small to large scales along $r_{1}$ at $x_{1} / d=2$ and along $r_{2}$ at $x_{1} / d=8$ also points from low to high $\mathcal{P}$ values. However, in the $x_{1} / d=2$ case, it does clearly point from high to low $\mathcal{P}$ values along the $r_{2}$ axis where the cascade appears forward, which does appear natural. We must leave the explanation of the non-intuitive relation between inverse cascade and scale-space $\mathcal{P}$ map for a future study which may involve all three directions in scale space and a better representation of coherent structures in the scale by scale energy balance.

The scale space map of $\Pi_{U}$ is mostly determined by the alignment of the mean velocity differences $\delta U_{i}$ with the gradient (in scale space) of $\left\langle\delta q^{2}\right\rangle$. Recall from fig. 16 that the distribution of $\left\langle\delta q^{2}\right\rangle$ in scale-space does not undergo significant changes between the two locations investigated. On the other hand the components of $\delta U_{i}$ can be expected to decrease significantly as the wake spreads and recovers towards $U_{\infty}$. In fact, $\delta U_{1}$ must remain positive for positive $r_{1}$ (as $U_{1}$ increases with $x_{1}$ ) and $\delta U_{2}$ must remain negative for positive $r_{2}$ due to the symmetry of the wake.

The results presented in this subsection illustrate how the present turbulent flow does not satisfy Kolmogorov's 1941 premises of equilibrium, local homogeneity and local isotropy even at separation length scales which correspond to the range where the energy spectra we presented have well-defined power laws with exponents close to $-5 / 3$. The nonlinear cascade occurs in a context where various other processes affecting turbulent kinetic energy are active, including production, advection and turbulent transport in physical space, correlations between fluctuating velocity differences and differences of pressure gradients, and linear interscale transfer by the mean flow. In the following subsection, we show how these processes balance when we average over planar orientations, thereby reducing the orientation averaged $\mathrm{KHMH}$ equation to $\Pi^{a} \approx-\varepsilon_{r}^{a} \approx-\varepsilon$ over a growing range of separations $r$ for further downstream centreline positions $x_{1} / d$ (the superscript $a$ indicates an average over planar orientation angles as illustrated in fig. 21). We have ascertained that, at $x_{1} / d=2$ and 8 on the centreline, $\varepsilon_{r}^{a}$ and $\varepsilon$ are very closely equal over the entire range $0 \leqslant r / d \leqslant 1.1$ of the figures discussed in the following subsection, so that if the quantities plotted in these figures were normalised by $\varepsilon$ then these figures would look effectively the same.

\subsection{Orientation Averaged KHMH Equation}

In fig. 29 we plot the planar orientation averaged terms of eq. (2.1) normalised by $\varepsilon_{r}^{a}$. These plots are shown for two centreline positions $x_{1} / d=2$ and $x_{1} / d=8$. At $r=0$ all terms vanish except $\varepsilon_{r}^{a}$ and $\mathcal{D}_{r}^{a}$ which balance exactly as can actually be shown analytically. At both positions, the viscous diffusion terms are negligible except at separations $r$ smaller than the Taylor length scale which is about $0.06 d$ at $x_{1} / d=2$ and $0.1 d$ at $x_{1} / d=8$. None of the other terms is negligible at separations $r$ larger than the Taylor length scale except $\Pi_{U}$ at the larger centreline distance. In fact, at $x_{1} / d=2$, all terms associated with inhomogeneity increase their contribution to eq. (2.1) as $r$ increases.

As the distance to the prism is increased, the contribution of $\Pi_{u}^{a}$ is considerably reduced (as already observed in fig. 28). It can also be seen in fig. 29 that even at the location furthest from the prism, the effect of the fluctuating pressure, included in $\mathcal{T}_{p}^{a}$, cannot be neglected, in particular at the largest separations. At the furthest of the two locations, it appears that the dependencies on $r$ of $\mathcal{A}^{a}$ and $\mathcal{T}_{u}^{a}$ mirror each other with a tendency for one to partly balance the other. This behaviour is consistent with the sweeping mechanism referred to in the previous subsection concerning $\mathcal{A}$ and $\mathcal{T}_{u}$ at $x_{1} / d=8$.

Finally, at both locations, we find a range of separations $r$ larger than the Taylor length 

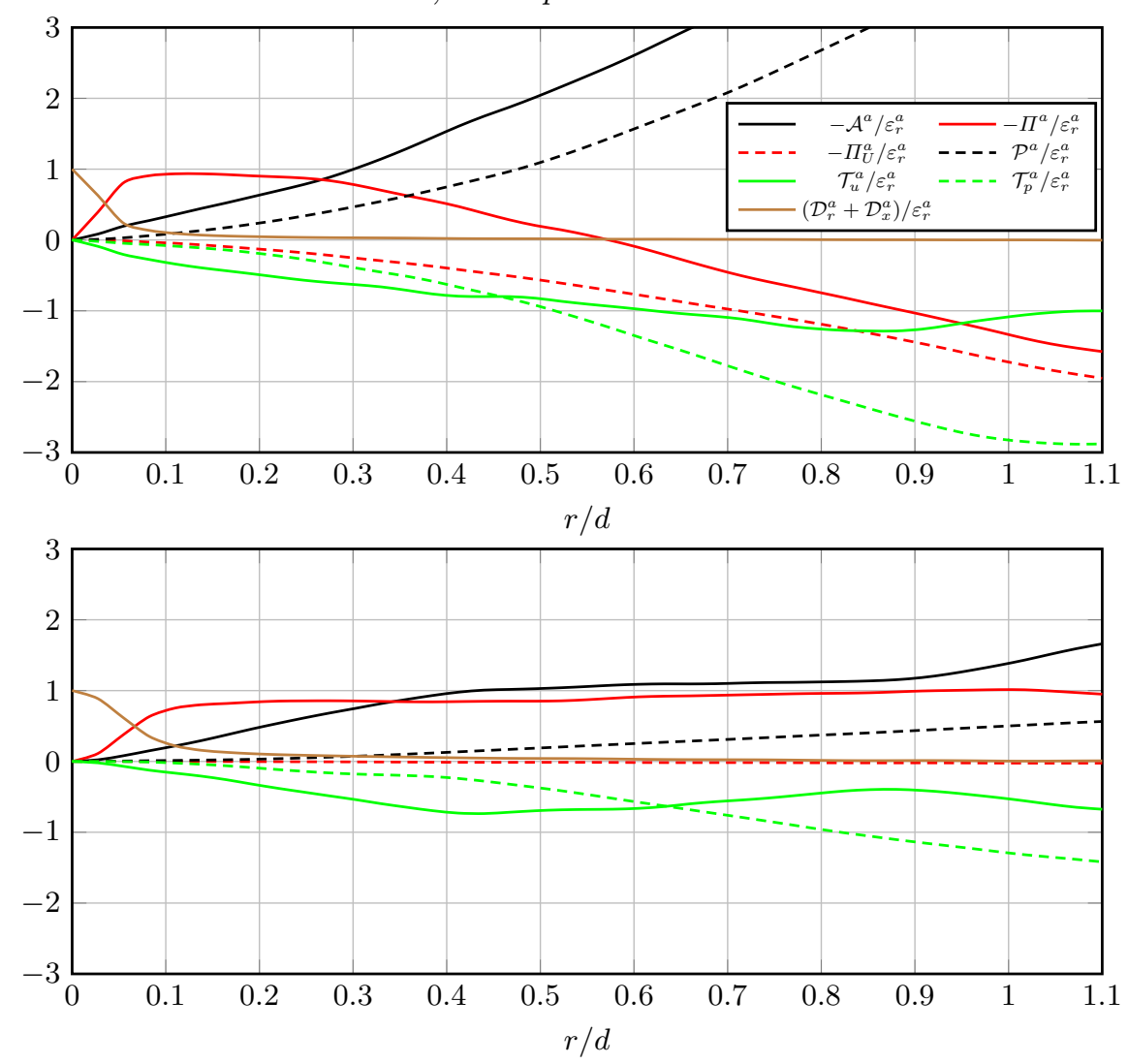

Figure 29: Orientation averaged terms of eq. (2.1) normalised by $\varepsilon_{r}^{a}$ at $x_{1} / d=2$ (top) and $x_{1} / d=8$ (bottom).

scale where the orientation averaged $\mathrm{KHMH}$ equation reduces to the following couple of approximate balances

$$
\Pi^{a}+\varepsilon_{r}^{a} \approx 0 \approx-\mathcal{A}^{a}+\mathcal{T}_{u}^{a}+\mathcal{T}_{p}^{a}-\left(\Pi_{U}^{a}-\mathcal{P}^{a}\right)
$$

This range of separations where $\Pi^{a} \approx-\varepsilon_{r}^{a} \approx-\varepsilon$ increases with increasing $x_{1} / d$ is clearly seen in fig. 30 where $\Pi^{a} / \varepsilon_{r}^{a}$ is plotted at four different locations on the centreline $\left(x_{1} / d=2,4,6,8\right)$. In fact, this range of separations covers one decade at $x_{1} / d=4,6,8$ and $\Pi^{a} \approx-\varepsilon_{r}^{a} \approx-\varepsilon$ appears to increase towards 1 with increasing $x_{1} / d$ in the range of separations where it is constant. The PIV measurements of Gomes-Fernandes et al. (2015) also found that $\Pi^{a}$ is approximately constant over a range of separations $r$ in the very near field of a different turbulent flow where the inhomogeneity terms of KHMH equation are also significant and in fact dominant. However their measurements indicated an apparently different value of $-\Pi^{a} / \varepsilon_{r}^{a}$, significantly larger than 1 . It is not clear whether their different value of $\Pi^{a} / \varepsilon_{r}^{a}$ is a result of the measurement limitations of their PIV and of the hypotheses that they were therefore forced to make to extract information on the $\mathrm{KHMH}$ equation from their measurements.

It is important to point out that the near constancy of $\Pi^{a}$ requires the inclusion of both it's in-plane $\left(\Pi_{r}^{a}\right)$ and out-of-plane $\left(\Pi_{z} \equiv \Pi_{3}\right)$ components (the angular contribution $\Pi_{\theta}^{a}=\frac{1}{2 \pi} \int_{0}^{2 \pi} \frac{1}{r} \frac{\partial\left\langle\delta u_{\theta} \delta q^{2}\right\rangle}{\partial \theta} \mathrm{d} \theta$ is zero). This is illustrated in fig. 31 where these two components are plotted as functions of $r$. Albeit small, the contribution of $\Pi_{3}^{a}$ is 


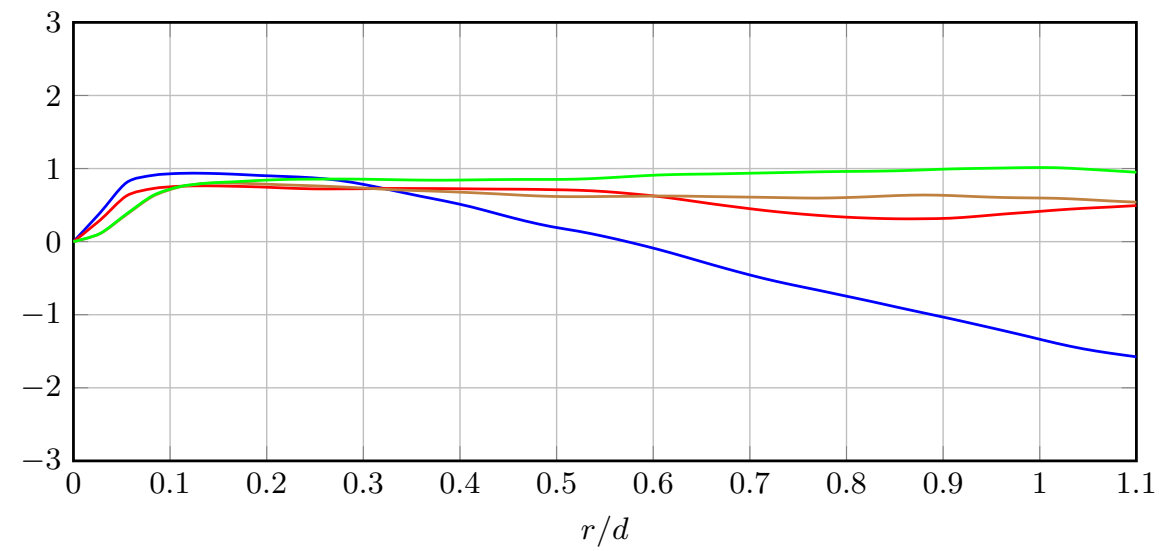

Figure 30: Orientation averaged non-linear inter-scale transfer $\Pi^{a}$ normalised by $-\varepsilon_{r}^{a}$ at four different locations along the centreline.

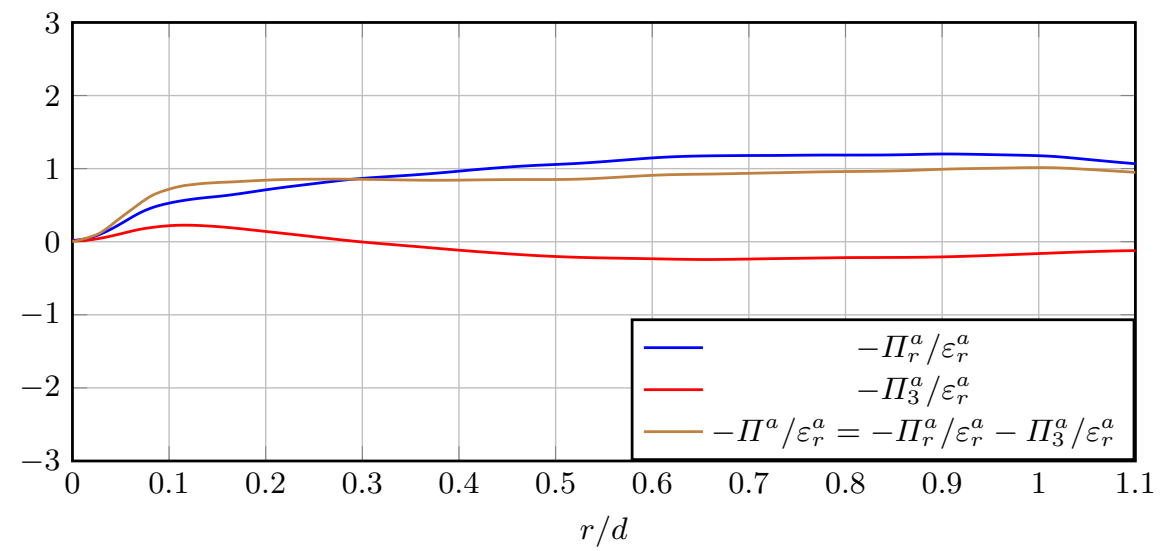

Figure 31: Orientation averaged in plane and out of plane components of $\Pi$ at $x_{1} / d=8$.

particularly relevant especially at the smaller separations, where both $\Pi_{r}^{a}$ and $\Pi_{3}^{a}$ are not constant but contribute to make $\Pi^{a}=\Pi_{r}^{a}+\Pi_{3}^{a}$ constant. It is likely that the inability of the experimental PIV of Gomes-Fernandes et al. (2015) to access all velocity field components may have affected their results.

As a final comment which echoes the last paragraph of section 4.1, the possibility remains that the inhomogeneity of the turbulence might be contributing to the constancy of $\Pi^{a}$.

\section{Conclusions}

The KHMH equation offers the possibility to study energy exchanges in any turbulent flow irrespective of its particular inhomogeneous or anisotropic character and suggests that well-defined energy-related laws may exist and may perhaps even be the same for a range of such flows. Remarkably, some of these laws seem to be similar to those predicted by the Kolmogorov theory of homogeneous turbulence but their underlying causes can of course not be explained by this theory.

In this paper we have investigated the turbulence in the very near wake of a square 
prism at $R e=3900$. Our results reveal that the power spectrum densities of the fluctuating velocity exhibit a near $-5 / 3$ slope over a wide range of frequencies on the centreline even though the flow is highly inhomogeneous and anisotropic. This slope is more evident at the location closest to the prism. We also find support for a Taylor frozen turbulence hypothesis. These spectra may therefore be the reflection of a Kolmogorovlike $r_{1}$-dependence of second order structure functions, even if this dependence is masked by coherent structure signatures.

The local inhomogeneity and local anisotropy in the region of the flow that we have focused on is made amply manifest by our study of all the terms in the fully generalised scale-by-scale energy balance, the Kármán-Howarth-Monin-Hill (KHMH) equation. The energy exchange processes caused by inhomogeneity are all active and some of them, specifically advection, turbulent transport in physical space and the term representing the effects of fluctuating pressure, are, along with the non-linear interscale cascade, the dominant processes in play. The strong dependence of all inhomogeneity terms on the orientation of the two-point separation vector shows that a conclusive study of the KHMH equation cannot be made by investigating pairs of points along a single orientation in a turbulent flow region such as the present one.

In spite of this extremely inhomogeneous and anisotropic setting and its related turbulent energy processes, the orientation averaged interscale transfer rate $\Pi^{a}$ is approximately independent of separation $r$ over a range of $r$ which increases with increasing $x_{1} / d$ from $x_{1} / d=2$ to at least $x_{1} / d=8$ on the centreline. Even more remarkably, this constant $\Pi^{a}$ tends towards $-\varepsilon_{r}^{a} \approx-\varepsilon$, i.e $\Pi^{a} \approx-\varepsilon_{r}^{a} \approx-\varepsilon$, as $x_{1} / d$ increases at least up to $x_{1} / d=8$. The computational domain of our DNS is not large enough to allow us an insight on what happens at normalised distances $x_{1} / d$ larger than 8 .

Even in the majority of cases (different values of $r$ and $x_{1}$ ) where the orientation averaged interscale transfer rate $\Pi^{a}$ is negative and the non-linear cascade is therefore forward on average, this overall cascade consists of a mix of forward and inverse nonlinear cascade behaviours in different orientations of the separation vector $\mathbf{r}$. These local, in scale space, inverse cascade behaviours coexist with energy spectra characterised by near $-5 / 3$ power laws. At $x_{1} / d=2$ where energy spectral power laws are best defined with exponents closest to $-5 / 3$, the inverse cascade is in the streamwise direction where the spectra are effectively evaluated. Such coexistence between $-5 / 3$ spectral scalings and an inverse non-linear cascade behaviour was already observed in Gomes-Fernandes et al. (2015) in the very near field of a different fully three-dimensional turbulent flow.

At the furthest downstream distance from the prism that our DNS can reliably reach, an approximate alignment appears to form between the scale space maps of the pressure term $\mathcal{T}_{p}$ and $\Pi$ on the one hand and between the advection $\mathcal{A}$ and the turbulent transport $\mathcal{T}_{u}$ on the other. The latter may suggest the appearance of a sweeping mechanism whereby the largest turbulent eddies transport small scale turbulence as it evolves.

Finally, our results reveal that the effects of the fluctuating pressure on the scale-byscale energy balance cannot be neglected, at least in the near field of a turbulent wake.

\section{Acknowledgements}

The authors acknowledge the EU support through the FP7 Marie Curie MULTISOLVE project (grant no. 317269) and the computational resources allocated in ARCHER HPC through the UKTC funded by the EPSRC grant no. EP/L000261/1 as well as the HPC resources provided by Imperial College on the cx2 facility. JCV also acknowledges the support of an ERC Advanced Grant (grant no. 320560). 


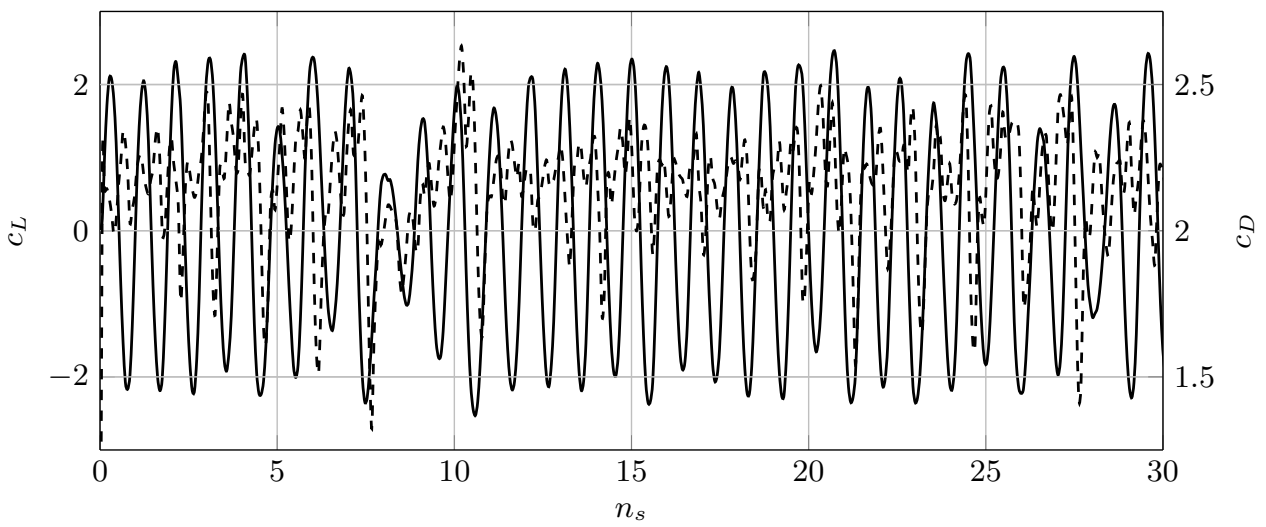

Figure 32: Time signals of $C_{L}$ (full line) and $C_{D}$ (dashed line) versus number of shedding periods $n_{s}=t f_{s}$.

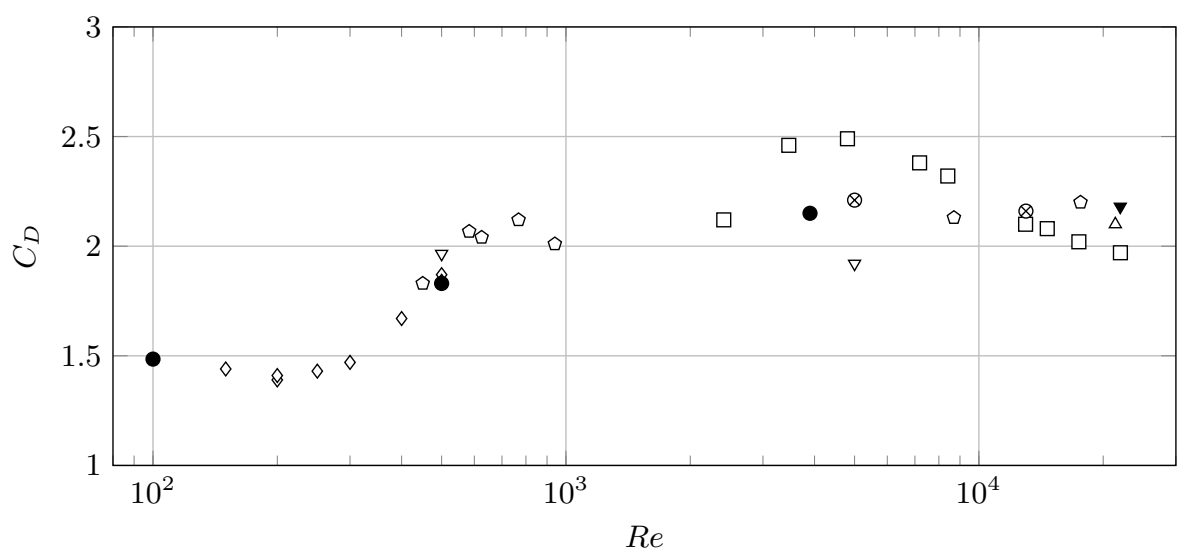

Figure 33: Mean drag coefficient at different Re. The symbols are the same as in fig. 3 .

\section{Appendix A. Further Comparisons with Experiments and DNS}

The shedding frequency $f_{s}$ was obtained by taking the power spectrum of the time signal of the lift coefficient $C_{L}$ (shown in fig. 32). The alternating peaks and valleys correspond to the instances in time when each vortex departs from the vortex formation region. The drag coefficient $C_{D}$ fluctuates with twice the shedding frequency (as seen in fig. 32 since each alternating vortex induces the same low pressure on the downstream face of the prism.

In figs. 33 to 35 we report, respectively, values of $C_{D}, C_{L}^{\prime}$ and $C_{D}^{\prime} / C_{L}^{\prime}$ at different $R e$. Despite the little availability of statistics for the force coefficients at $R e \sim \mathcal{O}\left(10^{3}\right)$, we find our results to fall within the experimental and numerical data of the listed references. For the present Reynolds number of 3900, the fluctuating lift coefficient has a standard deviation of $C_{L}^{\prime}=1.52$ and the drag coefficient has a mean value of $C_{D}=2.15$ with a standard deviation of $C_{D}^{\prime}=0.2$. Even though it appears that for the present $R e$ the $S t$ and $C_{D}$ are independent of $R e$, this appears not to be the case for both $C_{L}^{\prime}$ and $C_{D}^{\prime}$ as can be seen in figs. 34 and 35 . 


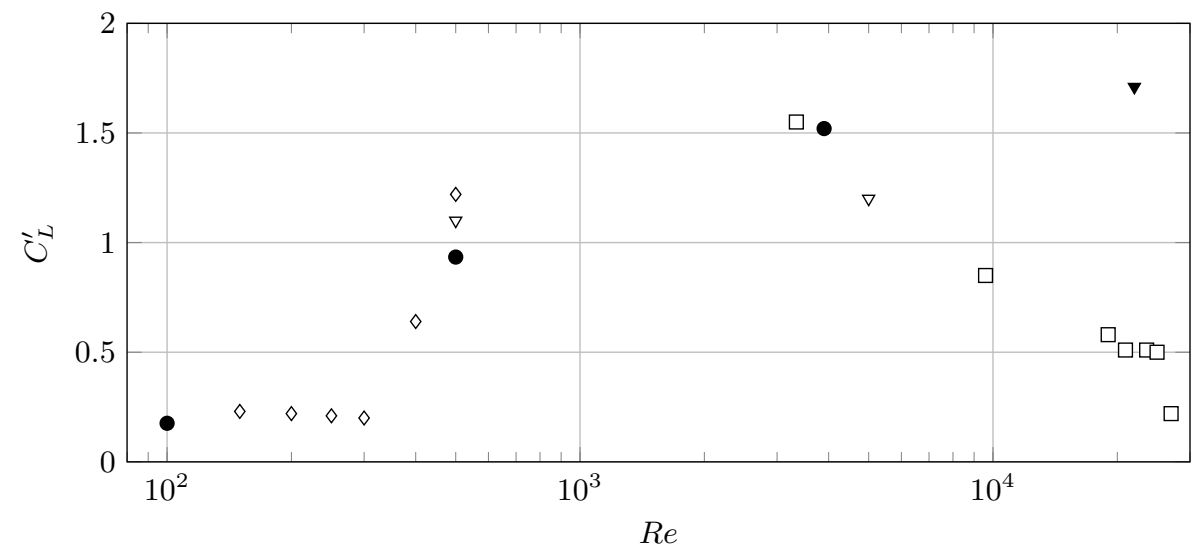

Figure 34: Root mean square of lift coefficient at different Re. The symbols are the same as in fig. 3 .

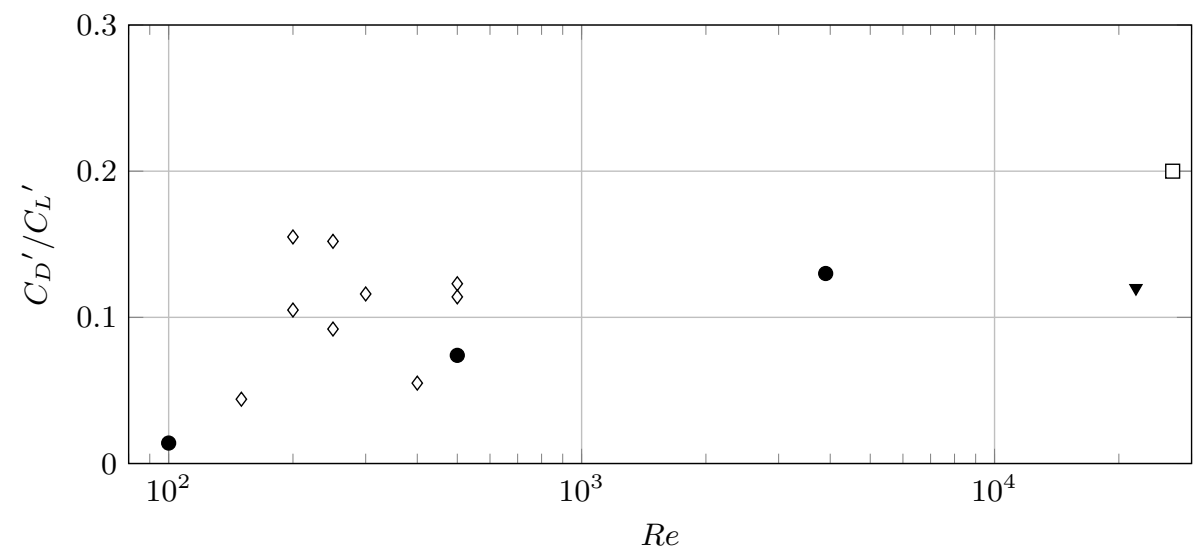

Figure 35: Ratio between the root mean square of drag and lift coefficients at different Re. The symbols are the same as in fig. 3 .

In fig. 36 the distribution of the mean spanwise averaged pressure coefficient

$$
C_{p}=\frac{P-P_{\infty}}{\frac{1}{2} \rho U_{\infty}^{2}}
$$

over the surface of the square is shown.

Good agreement is found when comparing the obtained distribution of $C_{p}$ along the surface with literature. Despite the scatter in the experimental results, we find that in our DNS the distribution of $C_{p}$ along the the back-face of the prism $(C D$ infig. 36) and on the top and bottom faces ( $B C$ and $D A$ in fig. 36) is within the data of Bearman \& Obasaju (1982), Norberg (1993), Chen \& Liu (1999) and Trias et al. (2015).

In fig. 37 the mean streamlines are shown in the vicinity of the prism (only the top half of the domain is shown). The large recirculation bubbles (connected by a saddle point at $x_{1} / d \approx 0.49, x_{2} / d \approx 0.6$ ) can be clearly identified above the prism's top surface and just downstream of it's backward side. In addition to these recirculation regions, two small separation bubbles exist on the top surface: an elongated one near the front-face and a much smaller one closer to the back-face. These separation bubbles have also been 


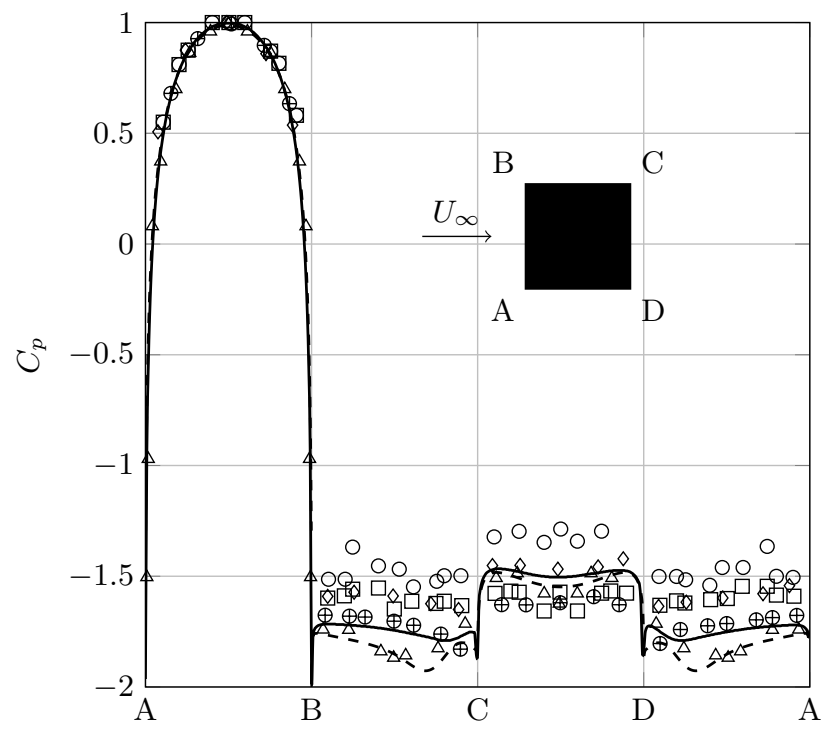

\begin{tabular}{|c|c|c|c|c|}
\hline$\square$ & $\begin{array}{l}\text { Chen \& Liu (1999) } \\
\quad(R e=2700)\end{array}$ & 0 & $\begin{array}{l}\text { Chen \& Liu (1999) } \\
\quad(R e=5300)\end{array}$ & $\begin{array}{l}\text { Norberg }(1993) \\
\quad(R e=5000)\end{array}$ \\
\hline$\Delta$ & $\begin{array}{c}\text { Bearman \& Obasaju (1982) } \\
(R e=5800) \\
\text { Present }\end{array}$ & -- & $\begin{array}{l}\text { Trias et al. }(2015) \\
\quad(R e=22000)\end{array}$ & $\begin{array}{c}\text { Mizota \& Okajima (1981) } \\
(R e=71400)\end{array}$ \\
\hline
\end{tabular}

Figure 36: Distribution of the spanwise averaged mean pressure coefficient $C_{p}$ over the sides of the prism. Experimental data are indicated by symbols, DNS by lines. The data from Norberg (1993) were taken from Sohankar et al. (1999). Bearman \& Obasaju (1982) only measured $C_{p}$ over the top half of the prism (from mid-point of $A B$ to the midpoint of $C D)$.

reported in Mizota \& Okaijima (1981), Sohankar (2006) and Trias et al. (2015) for flows at higher Reynolds numbers.

\section{Appendix B. Statistical Convergence}

Statistics were collected for about 30 shedding periods. Both single- and two-point statistics were then averaged in the spanwise direction. Traditionally, statistical convergence is assessed by computing a confidence interval which is computed from the standard deviation of the variable being sampled and knowledge regarding the number of independent samples in the dataset (related to the integral time scale, see George 1978; Benedict \& Gould 1996). As the wake is strongly affected by the shedding, computing the integral time scale is not trivial due to long correlations introduced by the coherent motion.

Figures 38 and 39 show the running averages of two-point statistics at the centreline of the wake for some combinations of mid-points and separations. Large variations are observed only in the first few shedding cycles. It can also be seen that the statistics of smaller separations fluctuate the least as the integral scales associated with the smallest separations (or smallest length scales) are indeed smaller, as noted in Valente \& Vassilicos (2015) where they compute the integral scales associated with each separation in order to determine (for each scale) the number of independent samples. 


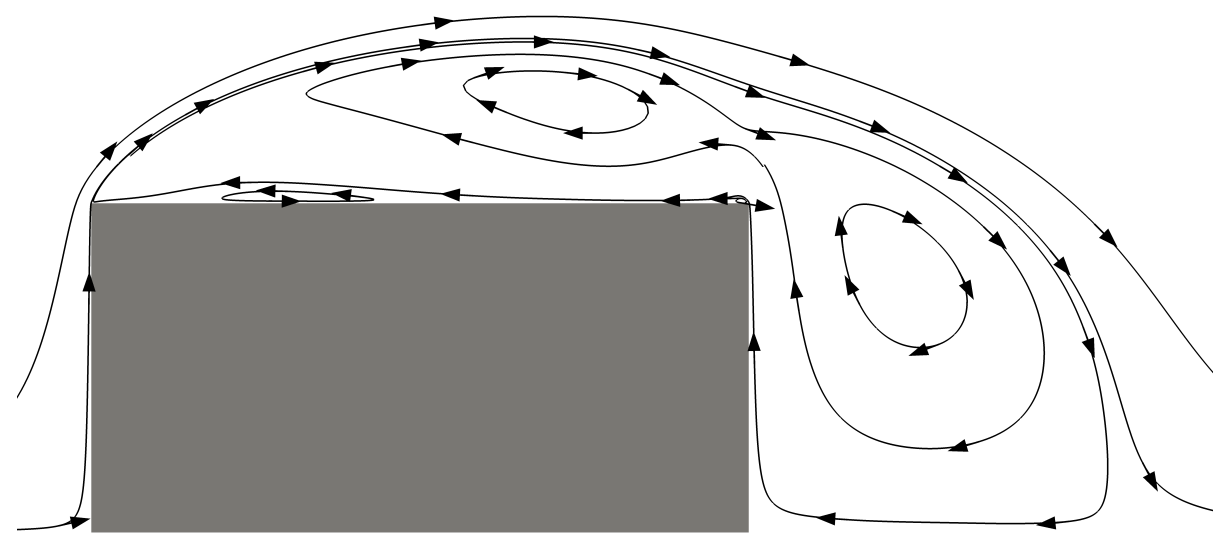

Figure 37: Mean streamlines in the vicinity of the prism. Only the top half of the domain is shown.

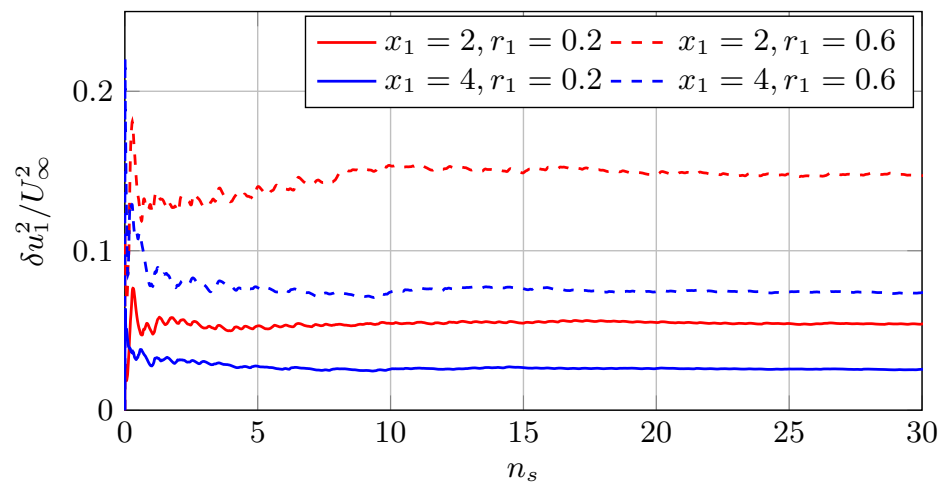

Figure 38: Running average of the spanwise averaged stream-wise second order structure function $\left\langle\delta u_{1}^{2}\right\rangle$ at three midpoints and for two different separations in the streamwise direction.

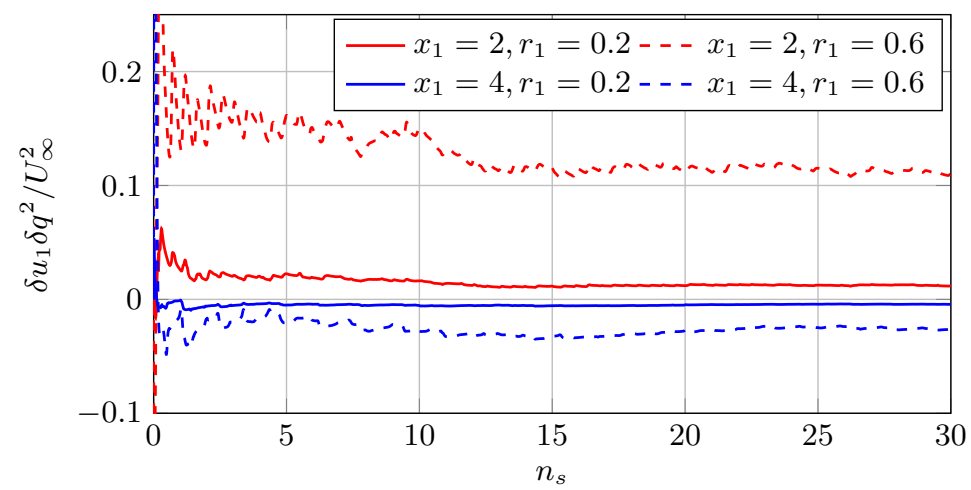

Figure 39: Running average of the spanwise averaged streamwise third order structure function $\left\langle\delta u_{1} \delta q^{2}\right\rangle$ at three midpoints and for two different separations in the streamwise direction. 

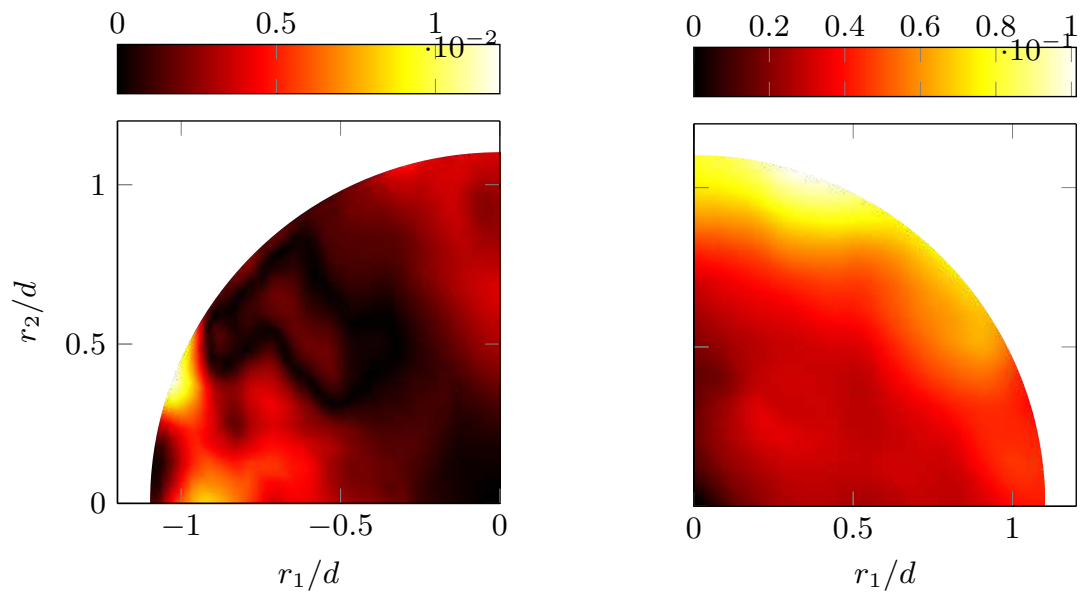

Figure 40: Distribution in scale space (for $r_{3}=0$ ) of the absolute value of time averaged transient term $\left|\mathcal{A}_{t}\right|$ normalised by the two-point dissipation $\varepsilon_{r}$ at two different locations on the centreline at $x_{1}=2 d$ (left) and $x_{1}=8 d$ (right).

A final assessment of the quality of the statistics collected is done by looking at the transient term of eq. (2.1) $\mathcal{A}_{t}$. In eq. (2.1) this term is written as $\frac{\partial\left\langle\delta q^{2}\right\rangle}{\partial t}$ since the averaging and time differentiation are assumed to commute. If one instead writes $\mathcal{A}_{t}$ as $\left\langle\frac{\delta q^{2}}{\partial t}\right\rangle$ then it will converge to zero with $T^{-1}$, where $T$ is the integration time. Checking how close $\mathcal{A}_{t}$ is to the expected value of zero is then also an indication of how well converged are the statistics.

In fig. 40 we plot the absolute value of $\left\langle\frac{\delta q^{2}}{\partial t}\right\rangle$ normalised by the two-point dissipation, highlighting it's relevance in the budget eq. (2.1). $\mathcal{A}_{t}$ remains at least one order of magnitude smaller than the dissipation at all orientations and locations considered.

\section{REFERENCES}

Antonia, R. A., Smalley, R. J., Zhou, T., Anselmet, F. \& Danaila, L. 2003 Similarity of energy structure functions in decaying homogeneous isotropic turbulence. Journal of Fluid Mechanics 487, 245-269.

Arslan, T., El Khoury, G. K., Pettersen, B. \& Andersson, H. I. 2012 Simulations of flow around a three-dimensional square cylinder using LES and DNS. In The Seventh International Colloquium on Bluff Body Aerodynamics and Applications, pp. 909-918.

Balay, S., Abhyankar, S., Adams, M. F., Brown, J., Brune, P., Buschelman, K., Dalcin, L., Eiskhout, V., Gropp, W. D., Kaushik, D., Knepley, M. G., Curfman Mcinnes, Lois, Rupp, K., Smith, B. F., Zampini, S., Zhang, H. \& Zhang, H. 2016 PETSc Users Manual. Tech. Rep. ANL-95/11 - Revision 3.7. Argonne National Laboratory.

Bearman, P. W. \& Obasaju, E. D. 1982 An experimental study of pressure fluctuations on fixed and oscillating square-section cylinders. Journal of Fluid Mechanics 119, 297.

Benedict, L. H. \& Gould, R. D. 1996 Towards better uncertainty estimates for turbulence statistics. Experiments in Fluids 22 (2), 129-136.

Bloor, M. S. \& Gerrard, J. H. 1966 Measurements on Turbulent Vortices in a Cylinder Wake. Proceedings of the Royal Society of London. Series A, Mathematical and Physical Sciences 294 (1438), 319-342.

Braza, M., Perrin, R. \& Hoarau, Y. 2006 Turbulence properties in the cylinder wake at high Reynolds numbers. Journal of Fluids and Structures 22 (6-7), 757-771. 
Cantwell, B. J. \& Coles, D. 1983 An experimental study of entrainment and transport in the turbulent near wake of a circular cylinder. Journal of Fluid Mechanics 136, 321.

Castro, I. P. 2016 Dissipative distinctions. Journal of Fluid Mechanics 788, 1-4.

Champagne, F. H., Harris, V. G. \& Corrsin, S. 1970 Experiments on nearly homogeneous turbulent shear flow. Journal of Fluid Mechanics 41 (01), 81.

Chen, J. M. \& Liu, C. H. 1999 Vortex shedding and surface pressures on a square cylinder at incidence to a uniform air stream. International Journal of Heat and Fluid Flow 20 (6), $592-597$.

Danaila, L., Krawczynski, J.F., Thiesset, F. \& Renou, B. 2012 Yaglom-like equation in axisymmetric anisotropic turbulence. Physica D: Nonlinear Phenomena 241 (3), 216-223.

Duchon, J. \& Robert, R. 1999 Inertial energy dissipation for weak solutions of incompressible Euler and Navier-Stokes equations. Nonlinearity 13 (1), 249-255.

Durão, D. F. G., Heitor, M. V. \& Pereira, J. C. F. 1988 Measurements of turbulent and periodic flows around a square cross-section cylinder. Experiments in Fluids 6 (5), 298-304.

Frisch, U. 1995 Turbulence: The Legacy of A. N. Kolmogorov. Cambridge University Press.

George, W. K. 1978 Processing of Random Signals. In Dynamic Flow Conference on Dynamic Measurements in Unsteady Flows (ed. Bengt Wulff Hansen), pp. 757-800. Dordrecht: Springer Netherlands.

Gomes-Fernandes, R., Ganapathisubramani, B. \& Vassilicos, J. C. 2015 The energy cascade in near-field non-homogeneous non-isotropic turbulence. Journal of Fluid Mechanics 771, 676-705.

Goto, S. \& VAssilicos, J. C. 2015 Energy dissipation and flux laws for unsteady turbulence. Physics Letters A 379 (16-17), 1144-1148.

Goto, S. \& Vassilicos, J. C. 2016 Local equilibrium hypothesis and Taylor's dissipation law. Fluid Dynamics Research 48 (2), 021402.

Hearst, R. J. \& Lavoie, P. 2014 Scale-by-scale energy budget in fractal element gridgenerated turbulence. Journal of Turbulence 15 (8), 540-554.

HILl, R. J. 1997 Applicability of Kolmogorov's and Monin's equations of turbulence. Journal of Fluid Mechanics 353, 67-81.

HiLl, R. J. 2001 Equations relating structure functions of all orders. Journal of Fluid Mechanics 434, 379-388.

Hill, R. J. $2002 a$ Exact second-order structure-function relationships. Journal of Fluid Mechanics 468, 317-326.

Hill, R. J. $2002 b$ The Approach of Turbulence to the Locally Homogeneous Asymptote as Studied using Exact Structure-Function Equations. Arxiv pp. 1-24, arXiv: 0206034.

Hu, J. C., Zhou, Y. \& Dalton, C. 2006 Effects of the corner radius on the near wake of a square prism. Experiments in Fluids 40 (1), 106-118.

IssA, R. I. 1986 Solution of the implicitly discretised fluid flow equations by operator-splitting. Journal of Computational Physics 62 (1), 40-65.

Klebanoff, P.S. 1955 Characteristics of Turbulence in a Boundary Layer with Zero Pressure Gradient. Tech. Rep.. National Advisory Comitee for Aeronautics Report 1247.

Kolmogorov, A. N. 1941a The local structure of turbulence in incompressible viscous fluid for very large Reynolds numbers. Doklady Akademiia Nauk SSSR 30.

Kolmogorov, A. N. $1941 b$ On the degeneration of isotropic turbulence in an incompressible viscous fluid. Doklady Akademiia Nauk SSSR 31.

Kolmogorov, A. N. 1941c Dissipation of Energy in the Locally Isotropic Turbulence. Doklady Akademiia Nauk SSSR 32.

Kraichnan, R. H. 1974 On Kolmogorov's inertial-range theories. Journal of Fluid Mechanics $62(02), 305$.

Kravchenko, A. G. \& Moin, P. 2000 Numerical studies of flow over a circular cylinder at $\mathrm{Re}_{\mathrm{D}}=3900$. Physics of Fluids 12 (2), 403.

Laizet, S., Nedić, J. \& Vassilicos, J. C. 2015 The spatial origin of $-5 / 3$ spectra in gridgenerated turbulence. Physics of Fluids 27 (6), 065115.

Laizet, S., Vassilicos, J. C. \& Cambon, C. 2013 Interscale energy transfer in decaying turbulence and vorticity-strain-rate dynamics in grid-generated turbulence. Fluid Dynamics Research 45 (6), 061408. 
Lee, M. \& Kim, G. 2001a A Study on the Near Wake of a Square Cylinder Using Particle Image Velocimetry (I) - Mean Flow (in Korean). Transactions of the Korean Society of Mechanical Engineers 25 (10), 1408-1416.

Lee, M. \& Kim, G. $2001 b$ A Study on the Near Wake of a Square Cylinder Using Particle Image Velocimetry (II) - Turbulence Characteristics (in Korean). Transactions of the Korean Society of Mechanical Engineers 25 (10), 1417-1426.

Lehmkuhl, O., Rodríguez, I., Borrell, R. \& Oliva, A. 2013 Low-frequency unsteadiness in the vortex formation region of a circular cylinder. Physics of Fluids 25 (8), 085109.

Lesieur, M. 2008 Turbulence in Fluids, 4th edn. Springer Netherlands.

Leslie, D. C. 1973 Developments in the theory of turbulence. Clarendon Press.

Lindiorg, E. 1996 A note on Kolmogorov's third-order structure-function law, the local isotropy hypothesis and the pressure-velocity correlation. Journal of Fluid Mechanics 326, 343-356.

LindBorg, E. 1999 Can the atmospheric kinetic energy spectrum be explained by twodimensional turbulence? Journal of Fluid Mechanics 388 (1999), S0022112099004851.

Lumley, J. L. 1965 Interpretation of Time Spectra Measured in High-Intensity Shear Flows. Phys. Fluids 8 (6), 1056.

Lyn, D. A., Einav, S., Rodi, W. \& PARK, J. H. 1995 A laser-Doppler velocimetry study of ensemble-averaged characteristics of the turbulent near wake of a square cylinder. Journal of Fluid Mechanics 304, 285.

Ma, X., Karamanos, G. S. \& Karniadakis, G. E. 2000 Dynamics and low-dimensionality of a turbulent near wake. Journal of Fluid Mechanics 410, 29-65.

Mathieu, J. \& Scott, J. F. 2000 An Introduction to Turbulent Flow. Cambridge University Press.

MсСомв, W. D. 2014 Homogeneous, Isotropic Turbulence. Oxford University Press.

Melina, G., Bruce, P. J. K. \& Vassilicos, J. C. 2016 Vortex shedding effects in gridgenerated turbulence. Physical Review Fluids 1 (4), 044402.

Mizota, T. \& Okajima, A. 1981 Experimental studies of time mean flows around rectangular prisms (in Japanese). In $J S C E$, , vol. 312, pp. 39-47.

Nedić, J., Tavoularis, S. \& Marusic, I. 2017 Dissipation scaling in constant-pressure turbulent boundary layers. Physical Review Fluids 2 (3), 032601.

Norberg, C. 1993 Flow around rectangular cylinders: Pressure forces and wake frequencies. Journal of Wind Engineering and Industrial Aerodynamics 49 (1-3), 187-196.

Obligado, M., Dairay, T. \& Vassilicos, J. C. 2016 Nonequilibrium scalings of turbulent wakes. Physical Review Fluids 1 (4), 044409.

Oвukнov, A. M. 1941 On the Energy Distribution in the Spectrum of a Turbulent Flow (in Russian). Doklady Akademiia Nauk SSSR 32 (1), 454-466.

ONG, L. \& WALLACE, J. 1996 The velocity field of the turbulent very near wake of a circular cylinder. Experiments in Fluids 20 (6), 441-453.

Pope, S. B. 2000 Turbulent Flows. Cambridge University Press.

Rogers, M. M. \& Moser, R. D. 1994 Direct simulation of a self-similar turbulent mixing layer. Physics of Fluids 6 (2), 903.

Sohankar, A. 2006 Flow over a bluff body from moderate to high Reynolds numbers using large eddy simulation. Computers \& Fluids 35 (10), 1154-1168.

Sohankar, A., Norberg, C. \& Davidson, L. 1999 Simulation of three-dimensional flow around a square cylinder at moderate Reynolds numbers. Physics of Fluids 11 (2), 288.

TAYlor, G. I. 1935 Statistical Theory of Turbulence. Proceedings of the Royal Society of London. Series A, Mathematical and Physical Sciences 23 (2), 421-444.

Tennekes, H. \& Lumley, J. L. 1972 A First Course in Turbulence. MiT Press.

Thiesset, F., Antonia, R. A. \& Danaila, L. 2013a Restricted scaling range models for turbulent velocity and scalar energy transfers in decaying turbulence. Journal of Turbulence 14 (3), 25-41.

Thiesset, F., Danaila, L. \& Antonia, R. A. 2011a Bilans énergétiques à chaque échelle prenant en considération le mouvement cohérent. In 20ème Congrès Français de Mécanique. Besançon, France: AFM.

Thiesset, F., Danaila, L. \& Antonia, R. A. $2013 b$ Dynamical effect of the total strain 
induced by the coherent motion on local isotropy in a wake. Journal of Fluid Mechanics 720, 393-423.

Thiesset, F., Danaila, L. \& Antonia, R. A. 2014 Dynamical interactions between the coherent motion and small scales in a cylinder wake. Journal of Fluid Mechanics 749 (April 2016), 201-226.

Thiesset, F., Danaila, L., Antonia, R. A. \& Zhou, T. $2011 b$ Scale-by-scale energy budgets which account for the coherent motion. Journal of Physics: Conference Series 318 (5), 052040 .

Trias, F. X., Gorobets, A. \& Oliva, A. 2015 Turbulent flow around a square cylinder at Reynolds number 22,000: A DNS study. Computers and Fluids 123 (22), 87-98.

Tsinober, A. 2009 An Informal Conceptual Introduction to Turbulence, 2nd edn. Springer.

Uberoi, M. S. \& Freymuth, P. 1969 Spectra of Turbulence in Wakes behind Circular Cylinders. Physics of Fluids 12 (7), 1359.

Valente, P. C. \& Vassilicos, J. C. 2015 The energy cascade in grid-generated nonequilibrium decaying turbulence. Physics of Fluids 27 (4), 045103.

Vassilicos, J. C. 2015 Dissipation in Turbulent Flows. Annual Review of Fluid Mechanics 47 (1), 95-114.

Voke, P. 1996 Flow Past a Square Cylinder: Test Case LES2. In Direct and Large Eddy Simulation II, , vol. 355-373, pp. 355-373.

Wissink, J. G. \& Rodi, W. 2008 Numerical study of the near wake of a circular cylinder. International Journal of Heat and Fluid Flow 29 (4), 1060-1070.

WyngaARD, J. C. \& Clifford, S. F. 1977 Taylor's Hypothesis and High-Frequency Turbulence Spectra. Journal of the Atmospheric Sciences 34 (6), 922-929.

Yasuda, T. \& VAssilicos, J. C. 2017 Inhomogeneous energy cascade in periodic turbulence (in preparation) .

Zhou, Y. \& Antonia, R. A. 1992 Convection velocity measurements in a cylinder wake. Experiments in Fluids 13, 63-70. 